Check for updates

Cite this: RSC Adv., 2019, 9, 38209

Received 12th September 2019 Accepted 4th November 2019

DOI: $10.1039 / c 9 r a 07342 f$

rsc.li/rsc-advances

\section{An insight into biomimetic 4D printing}

\author{
Nand Jee Kanu, (D) *a Eva Gupta, (DD ${ }^{\text {b }}$ Umesh Kumar Vates (D) \\ and Gyanendra Kumar Singh (D) ${ }^{d}$
}

4D printed objects are indexed under additive manufacturing (AM) objects. The 4D printed materials are stimulusresponsive and have shape-changing features. However, the manufacturing of such objects is still a challenging task. For this, the designing space has to be explored in the initial stages, which is lagging so far. This paper encompasses two recent approaches to explore the conceptual design of 4D printed objects in detail: (a) an application-based modeling and simulation approach for phytomimetic structures and (b) a voxel-based modeling and simulation approach. The voxel-based modeling and simulation approach has the enhanced features for the rapid testing (prior to moving into design procedures) of the given distribution of such 4D printed smart materials (SMs) while checking for behaviors, particularly when these intelligent materials are exposed to a stimulus. The voxel-based modeling and simulation approach is further modified using biexponential expressions to encode the time-dependent behavior of the bio-inspired 4D printed materials. The shape-changing materials are inspired from biological objects, such as flowers, which are temperaturesensitive or touch-sensitive, and can be 4D printed in such a way that they are encrypted with a decentralized, anisotropic enlargement feature under a restrained alignment of cellulose fibers as in the case of composite hydrogels. Such plant-inspired architectures can change shapes when immersed in water. This paper also outlines a review of the 4D printing of (a) smart photocurable and biocompatible scaffolds with renewable plant oils, which can be a better alternative to traditional polyethylene glycol diacrylate (PEGDA) to support human bone marrow mesenchymal stem cells (hMSCs), and (b) a biomimetic dual shape-changing tube having applications in biomedical engineering as a bioimplant. The future applications would be based on these smart and intelligent materials; thus, it is important to modify the existing voxel-based modeling and simulation approach and discuss efficient printing methods to fabricate such bio-inspired materials.
${ }^{a}$ S. V. National Institute of Technology, Surat, India. E-mail: nandssm@gmail.com; Tel: +919579248080

${ }^{b}$ Amity University, Uttar Pradesh, India

${ }^{c}$ Amity University, Uttar Pradesh, India
${ }^{d}$ Federal Technical and Vocational Education and Training Institute, Addis Ababa, Ethiopia

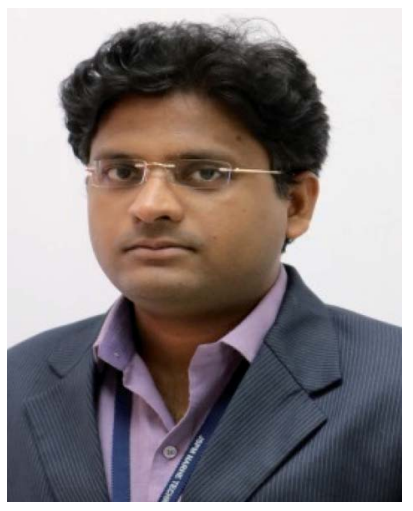

Nand Jee Kanu is a Research Scholar at the S. V. National Institute of Technology, Gujarat, India (an Institute of National Importance). He is pursuing Ph.D. in Mechanical Engineering. He has received his M.Tech. degree in Mechanical Engineering with a specialization in CAD/CAM. He has over five years of experience in Industry and Academics. His research interests include the interdisciplinary field of materials science, but they are not limited to smart materials such as biomimetic $4 D$ printed materials, self-healing composites and biofunctional nanofibers as well as structural health monitoring for damage detection and characterization strategies for engineering structures.

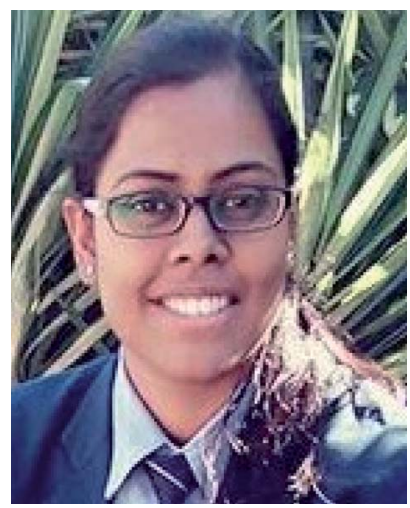

Eva Gupta is a Research Scholar at Amity University, Noida, India (an Institute of National Importance), and she is pursuing Ph.D. in Electrical Engineering. She received her M.Tech. degree in power distribution with a specialization in smart grids from the University of Petroleum and Energy Studies, Uttarakhand, India. She has over two years of experience in Industry and Academics. Her research interests include the interdisciplinary field of materials science, but they are not limited to smart materials such as $4 D$ printed materials, self-healing composites and biofunctional nanofibers as well as smart grids in electrical distribution networks for green and reliable operations. 


\section{Introduction}

The concept of $4 \mathrm{D}$ printed (4DP) smart materials was coined by Tibbits in the year $2013^{\mathbf{1}}$ in his speech at the MIT (USA) conference. Such stimuli-responsive materials have one extra dimension (fourth dimension) as compared to 3D printed materials. These intelligent materials were modeled using a bi-exponential mathematical model to predict their fourth dimension. ${ }^{2}$ Several methods have been introduced for the printing of such smart materials such as laser-assisted bioprinting, ${ }^{3}$ fused deposition modeling, and other methods common to the $3 \mathrm{D}$ printing of CAD models. ${ }^{1}$ The materials for such bio-inspired structures are selected on the basis of their responses to environmental and temporal stimuli. ${ }^{4}$ In the case of thermo-responsive smart materials, the shape memory effect (SME) is the key mechanism to drive deformation. Nowadays, researchers are freely using such shape memory alloys for the ease in the printing of such smart bio-inspired materials. The shape memory polymers (SMPs) are programmed above their glass transition temperature $\left(T_{\mathrm{g}}\right)$ and further cooled to set their temporary shape, which is free of any external loading. ${ }^{5-9}$ The $4 \mathrm{D}$ printed (4DP) smart material recovers its permanent shape once the temperature is raised above its $T_{\mathrm{g}}$.

The bio-inspired 4DP materials such as hydrogels (which can expand to twice their original volume) are capable of changing their shapes when immersed in water. The hydrogel can be immersed to absorb water until it is saturated. The swelling of the hydrogel is controlled by varying the temperature of the aqueous environment. ${ }^{10-14}$ The smart 4DP shape-folding structures can change shape under heat and light. ${ }^{15}$ When light was incident over certain parts of a polymer gel block, the part swelled due to the infiltration of chromophores (photo-responsive) at the illuminated site. ${ }^{16}$ By the virtue of anisotropy, the 4DP materials bend when exposed to UV rays. ${ }^{17}$ The electro-responsive $4 \mathrm{DP}$ materials also attracted attention after the introduction of the silicon elastomer and ethanol-based artificial muscle. In particular, when heat is generated after the passage of an electric current, ethanol evaporates and

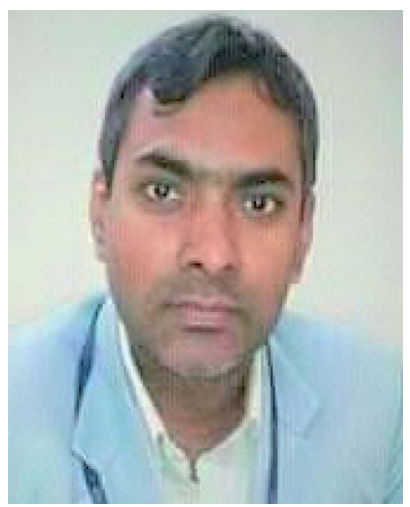

Umesh Kumar Vates is working as an Associate Professor in the Department of Mechanical Engineering, Amity University, Noida, India. He has completed his Ph.D. in Mechanical Engineering from IIT (ISM) Dhanbad, India (an Institute of National Importance). He has over two decades of experience in Industry and Academics. He holds a lifetime membership of the Indian Society of Technical Education. His research interests include the interdisciplinary field of materials science, but they are not limited to smart materials such as biomimetic $4 D$ printed materials, self-healing composites and biofunctional nanofibers as well as the modeling and optimization of modern manufacturing processes. the volume of the $4 \mathrm{DP}$ materials increases thereafter due to the increase in the volume of ethanol. ${ }^{18}$ An electric current is also used to absorb or desorb water to control the volume of the matrix of the 4DP materials. ${ }^{19}$ The $4 \mathrm{DP}$ materials are also found to respond to magnetic fields. One example is the magnetic nanoparticleincorporated microgripper, which was printed using hydrogels. ${ }^{13}$

The 4DP materials have been used as smart materials in drug delivery using 4DP containers, stent fabrication, splint fabrication and others. ${ }^{\text {20-31 }}$ The 4DP containers (printed using photolithography) are used to release pharmaceutical drugs at targeted locations when triggered by environmental stimuli. The stents are programmed in such a way that they can return to their initial shape after implantation. ${ }^{27,28}$ The $4 \mathrm{DP}$ biomedical devices, such as splints, can be accommodated for growth over time. ${ }^{29-31}$

There is a need for heat-sensitive 4DP smart materials in the human body that can change their shape with time. The biological activities in human bodies are based on response-guided transmissions involving enzymes, glucose, proteins, etc. such as the regulation of blood glucose (sugar level) through insulation (which is secreted by islet cells). ${ }^{32,33}$ Nowadays, the trending research topics are the development of $4 \mathrm{D}$ printed (1) glucoseresponsive materials having potential applications in observing the glucose level and delivering insulin ${ }^{\mathbf{3 4 2}}$ and (2) enzyme-responsive materials having potential applications in drug delivery ${ }^{\mathbf{4 3 - 5 0}}$ and tissue regeneration. ${ }^{51,52}$ Through this review article, five case studies have been discussed in the next few sections on the recent advances in the modeling and simulation as well as the printing of biomimetic 4D materials.

\section{Case studies}

2.1 Mechanics of 4D printed phytomimetic materials using the application-based modeling framework for conceptual design

The discrete geometry of bilayer materials was modeled into the continuous plates having nonuniform thicknesses using the

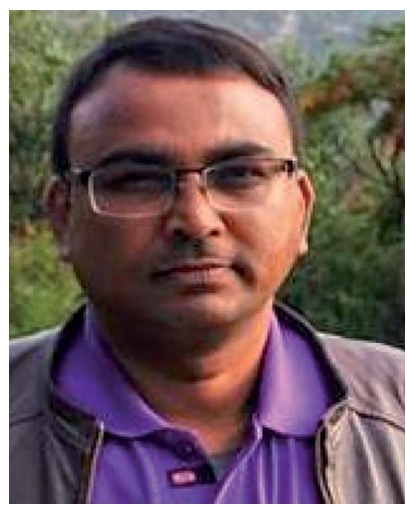

Gyanendra Kumar Singh is working as a Faculty member at the Federal Technical and Vocational Education and Training Institute, Ethiopia. He has completed his Ph.D. in Mechanical Engineering from the M. N. National Institute of Technology, Allahabad, India (an Institute of National Importance). He has over two decades of experience in Academics. He holds a lifetime membership of the Indian Society of Technical Education. His research interests include the interdisciplinary field of materials science, but they are not limited to smart materials such as $4 D$ printed materials, selfhealing composites and biofunctional nanofibers as well as the modeling and optimization of modern manufacturing processes. 
application-based numerical approach for the smart 4D printed phytomimetic structures. The final shapes were observed after subjecting the materials to specified swell anisotropies. ${ }^{53}$ The predictions of the model were in good agreement with experimental data sets. ${ }^{54}$

The framework for the conceptual design was based on the elasticity of the swelling active and passive bilayers. The energy function for any active or passive monolayer was described using eqn (1). ${ }^{55-57}$

$$
E_{\mathrm{ML}}=\frac{1}{2} \int_{U}\left[\frac{h}{4}\left\|a_{\mathrm{r}}^{-1} a_{\mathrm{c}}-I\right\|_{e}^{2}+\frac{h^{3}}{12}\left\|a_{\mathrm{r}}^{-1}\left(b_{\mathrm{c}}-b_{\mathrm{r}}\right)\right\|_{e}^{2}\right] \sqrt{\operatorname{det} a_{\mathrm{r}}} \mathrm{d} x \mathrm{~d} y
$$

The curvilinear coordinates, $(x, y) \in U$, was used to represent the mid-surface of the material in eqn (1). The thickness, $h$, was expressed in relation to the rest metric, $\left(a_{\mathrm{r}}\right)$, and the geometry of the mid-surface, $a_{\mathrm{c}}=(d \vec{m})^{T} d \vec{m}$, as $\left\|a_{r}^{-1} a_{c}-I\right\| \leq h$. In eqn (1), the geometry of the mid-surface was expressed in the second fundamental form using $b_{\mathrm{c}}=-(d \vec{m})^{T} d \vec{n}=-(d \vec{n})^{T} d \vec{m}$, and the rest second fundamental metric $\left(b_{\mathrm{r}}\right)$.

The above formulation was further expressed as per the classical laminated plate theory $(\mathrm{CLPT})^{58}$ for active and passive bilayers, as shown in eqn (2). ${ }^{53}$

$$
a_{\mathrm{r}}=\frac{1}{2}\left(a_{\mathrm{r} 1}+a_{\mathrm{r} 2}\right) ; b_{\mathrm{r}}=\frac{3}{4 h}\left(a_{\mathrm{r} 1}-a_{\mathrm{r} 2}\right)
$$

In eqn (2), the growth patterns of the active (top) and passive (bottom) layers were expressed using symbols $a_{\mathrm{r} 1}$ and $a_{\mathrm{r} 2}$, respectively. The common mid-surface was assumed for both layers. The essential elastic energy of the bilayer material was calculated by substituting the values of $a_{\mathrm{r}}$ and $b_{\mathrm{r}}$ in eqn (1). ${ }^{55}$ The framework thus could be used to analyze the equilibrium shapes within the given boundary conditions, material parameters, and associated first and second general forms of the growth or swell tensors.

The mid-surface of the plate was meshed using triangular $(T)$ elements. ${ }^{57,59}$ The overall deformation energy of the plate was described using a modified form of eqn (1), as shown in eqn (3).

$$
\begin{aligned}
E_{\mathrm{ML}} \approx & \frac{1}{2} \sum_{T \in \Gamma}\left[\frac{h_{T}}{4}\left\|\left(a_{\mathrm{r}}\right)_{T}^{-1}\left(a_{\mathrm{c}}\right)_{T}-I\right\|_{e, T}^{2}\right. \\
& \left.+\frac{h_{T}^{3}}{12}\left\|\left(a_{\mathrm{r}}\right)_{T}^{-1}\left[\left(b_{\mathrm{c}}\right)_{T}-\left(b_{\mathrm{r}}\right)_{T}\right]\right\|_{e, T}^{2} \frac{\sqrt{\operatorname{det}\left(a_{\mathrm{r}}\right)_{T}}}{2}\right]
\end{aligned}
$$

The design of the print path was further converted to a gradually changing thickness discipline and a swell metric tensor. The procedure to convert the print path was described using eqn (4) and (5) for the uniform density and growth field.

$$
\tilde{\rho}(x)=\sum_{i=1}^{N_{\mathrm{f}}} \int_{0}^{1} \zeta\left(x-p_{i}(t), p_{i}^{\prime}(t) ; \sigma\right)\left|p_{i}^{\prime}(t)\right| \mathrm{d} t
$$

In eqn (4), $N_{\mathrm{f}}, p_{i}(t), \zeta(x, y ; \sigma)$, and $\sigma$ were used to express the uniform splines, parametric equation of the $i$ th filament, the leveling kernel, and the scalar parameter to control thickness, respectively. The parametric equation, $p_{i}(t) \in \mathbb{R}^{2}$, was operated by varying $t$ in the range of 0 and 1 . The tangent vector, $p_{i}^{\prime}(t)$, was used to express the direction of growth, as shown in eqn (5).

$$
p_{\mathrm{c}}(x)=\sum_{i=1}^{N_{\mathrm{f}}} \int_{0}^{1} \zeta\left(x-p_{i}(t), p_{i}^{\prime}(t) ; \sigma\right)\left|p_{i}^{\prime}(t)\right| \mathrm{d} t
$$

In eqn (5), normalized $p_{\mathrm{c}}$ was used to obtain the unit vector. The growth pattern was analyzed while fixing the rest metric at all points of the layer as per the direction of growth (usually referred to as eigenvectors) and factors of growth (usually referred to as eigenvectors), as shown in the set of eqn (6).

$$
\left.\begin{array}{l}
a_{\mathrm{r} 1}(x)=R_{1}^{T}(x) \Lambda_{1}^{2} R_{1}(x) \\
a_{\mathrm{r} 2}(x)=R_{2}^{T}(x) \Lambda_{2}^{2} R_{2}(x) \text { in general } l \in[1,2]
\end{array}\right\}
$$

where, $\Lambda_{\mathrm{l}}=I+\mu_{\mathrm{l}}\left(\begin{array}{cc}s_{\mathrm{p}} & 0 \\ 0 & s_{\mathrm{t}}\end{array}\right)$, and $R_{\mathrm{l}}(x)=\left(\hat{p}_{\mathrm{c}, \mathrm{l}}(x) \hat{p}_{\mathrm{c}, 1}^{\perp}(x)\right)$.

In eqn (6), $l$ and $\mu_{1}$ were used to represent the active (top as 2) and passive (bottom as 1) layers, and the corresponding scaling factors, respectively, whereas $s_{\mathrm{p}}$ and $s_{\mathrm{t}}$ were used to represent the factors of growth in the parallel and transverse directions to the filament, respectively. ${ }^{53}$ The simulation results were validated with experimental data sets ${ }^{54}$ for all artificial and natural shapes, and thus, this framework could be used to predict the shape-changing events of the 4D printed smart bilayer materials.

\subsection{Modeling and simulation of the 4D printed smart materials using the voxel-based modeling framework for conceptual design}

The $4 \mathrm{D}$ printed (4DP) smart materials having shape-changing capabilities were modeled successfully using the voxel-based modeling and simulation method. ${ }^{60}$ In such modeling framework, it is necessary to model the real nature of matter along with the actual modeled distribution (MD). ${ }^{61-64}$ The most important thing that must be taken into consideration is modeling for the sensitivity towards stimuli, such as light and heat, in terms of triggering their shape-changing features as it happens in the case of flowers. ${ }^{65-73}$ The continuum mechanics in such cases were started with the rough design space solution, progressed through the discretization of the model, and then ended with the computation of the voxelized object deformation. ${ }^{60}$ The mass-spring modeling was considered fundamental to the concept used behind the definition of the design space and discretization. The voxels were connected from their centers in 3D beams. These beams were connected from their centers and thus, in a frame, they held the matter together. In this way, the entire deformation was taken care of in the geometry. The linear voxel was modeled as a uniform isotropic element. The Young's modulus $(E)$ and shear modulus $(G)$ were thus required to specify the mechanical behavior, along with the addition of the stimulus-responsive behavior. To accomplish the task of mapping the beam material with voxels, the properties of the beam materials were inherited from the pairs of voxels to which they were connected. As a matter of fact, two voxels were made of dissimilar materials, and the material properties were described using eqn (7). ${ }^{60}$ 


$$
E_{\mathrm{c}}=\frac{2 E_{1} E_{2}}{E_{1}+E_{2}}, \quad G_{\mathrm{c}}=\frac{2 G_{1} G_{2}}{G_{1}+G_{2}}
$$

The 3D Euler-Bernoulli beam concepts were used for the computations of deformations to incorporate the properties (such as improved axial, bending, and twisting resistances) in the beams, as shown in eqn (8). ${ }^{60}$

$$
I_{y y}=I_{z z}=I=\frac{b h^{3}}{12}=\frac{l^{4}}{12}, \quad J_{x x}=J=\frac{b h\left(b^{2}+h^{2}\right)}{12}=\frac{l^{4}}{6}
$$

In eqn (8), the properties of the cross-section of the beam were assumed along the $X$-axis, and ' ' ' was used to represent the size of the voxel. The torsional rigidity was characterized using the moment of inertia of the beam, as shown in the abovementioned equation. The cross sectional area, $A=l^{2}$, has to be substituted in the above eqn (8).

The direct stiffness method $\left(\bar{K}_{\mathrm{e}}\right)^{74}$ was used to calculate the degrees of freedom (DOFs; in this case, each node had six DOFs in the global coordinate system, as shown in eqn $\left.(9)^{60}\right)$ and the deformed shape.

$$
U_{i}=\left[\begin{array}{llllll}
x_{i} & y_{i} & z_{i} & \theta_{x i} & \theta_{y i} & \theta_{z i}
\end{array}\right], \quad K U=F
$$

$$
M^{\prime}\left(\begin{array}{lll}
x & y & z
\end{array}\right)=\phi(M)=X \vec{e}_{x}+\left[\begin{array}{l}
u_{x}(X) \\
u_{y}(X) \\
u_{z}(X)
\end{array}\right]+\Lambda(X)\left[\begin{array}{l}
0 \\
Y \\
Z
\end{array}\right]
$$

In eqn (10), the function $M^{\prime}\left(\begin{array}{lll}x & y & z\end{array}\right)$ was used to represent the point in the deformed state, and $u_{i}(X)$ was used to describe the translational DOFs along the $i$-axis at the location of $X$. In the above equation, $\Lambda(X)$ was used as the function of $\theta_{x}(X), \theta_{y}(Y), \theta_{z}(Z)$, where $\theta_{i}(X)$ was used as a general representation of the rotational DOF about the $i$-axis at the location of $X$. The general form of $\Lambda(X)$ is shown using eqn (11). ${ }^{60}$

$$
\Lambda(X)=R_{z}\left(\theta_{z}(X)\right) R_{y}\left(\theta_{y}(X)\right) R_{x}\left(\theta_{x}(X)\right)
$$

In eqn (11), $R_{i}$ was used for the rotation about the $i$-axis. The values of the DOFs were known at nodes 1 and 2, as discussed in eqn (10). Thus, the shape functions were used to find their values at any location of $X$, as discussed using the set of eqn (12). ${ }^{60}$

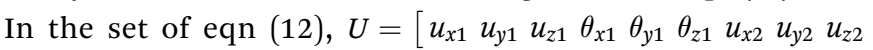
$\left.\begin{array}{lll}\theta_{x 2} & \theta_{y 2} & \theta_{z 2}\end{array}\right]^{\mathrm{T}}$, and the values of the shape functions are listed in the set of eqn (13). ${ }^{60}$

$$
\begin{aligned}
{\left[\begin{array}{l}
u_{x} \\
u_{y} \\
u_{z} \\
\theta_{x}
\end{array}\right] } & =\left[\begin{array}{cccccccccccc}
N_{1} & 0 & 0 & 0 & 0 & 0 & N_{2} & 0 & 0 & 0 & 0 & 0 \\
0 & N_{3} & 0 & 0 & 0 & -N_{4} & 0 & N_{5} & 0 & 0 & 0 & -N_{6} \\
0 & 0 & N_{3} & 0 & N_{4} & 0 & 0 & 0 & N_{5} & 0 & N_{6} & 0 \\
0 & 0 & 0 & N_{1} & 0 & 0 & 0 & 0 & 0 & N_{2} & 0 & 0
\end{array}\right] U \\
\theta_{y} & =-\frac{\mathrm{d} u_{z}}{\mathrm{~d} X} \\
\theta_{z} & =\frac{\mathrm{d} u_{y}}{\mathrm{~d} X}
\end{aligned}
$$

The entire system of equations was formulated under the framework shown in eqn (9). The global stiffness matrix is shown using the $U$ matrix $\left(U=\left[U_{i} \ldots U_{n}\right]^{T}\right.$, where $n$ is the number of voxels), and $F$ was used to represent $6 n$ vector having values of boundary conditions for force and moments, along with rotations and displacements.
The above eqn (10)-(13) were expressed in the general coordinate system (GCS) and during implementation, they were allowed to map the nodes in the global coordinate system (GCS). The voxels had eight vertices at their corners. In order to capture the deformed shape of the voxels, the authors ${ }^{60}$ suggested increasing the number of vertices after re-meshing their

$$
\left.\begin{array}{rl}
N_{1}(X) & =-\frac{1}{L}\left(X-X_{2}\right), \quad N_{2}(X)=\frac{1}{L}\left(X-X_{1}\right), \quad N_{3}(X)=1-\frac{3 \bar{X}^{2}}{L^{2}}+\frac{2 \bar{X}^{3}}{L^{3}} \\
N_{4}(X) & =\bar{X}\left(-1+\frac{2 \bar{X}}{L}-\frac{\bar{X}^{2}}{L}\right), N_{5}(X)=\frac{\bar{X}^{2}}{L^{2}}\left(3-\frac{2 \bar{X}}{L}\right), N_{6}(X)=\frac{\bar{X}^{2}}{L}\left(1-\frac{\bar{X}}{L}\right) \\
\bar{X} & =X-X_{1}
\end{array}\right\}
$$

The beam was mapped along the $X$-axis (Fig. 1) and its centre line was extended from node 1 to node 2 , as shown in eqn $(10) .^{75}$ surfaces. The vertices of the voxels were stored and extracted thereafter. The authors ${ }^{60}$ further specified the influence zone for each beam to find its deformation. For this, they have calculated 

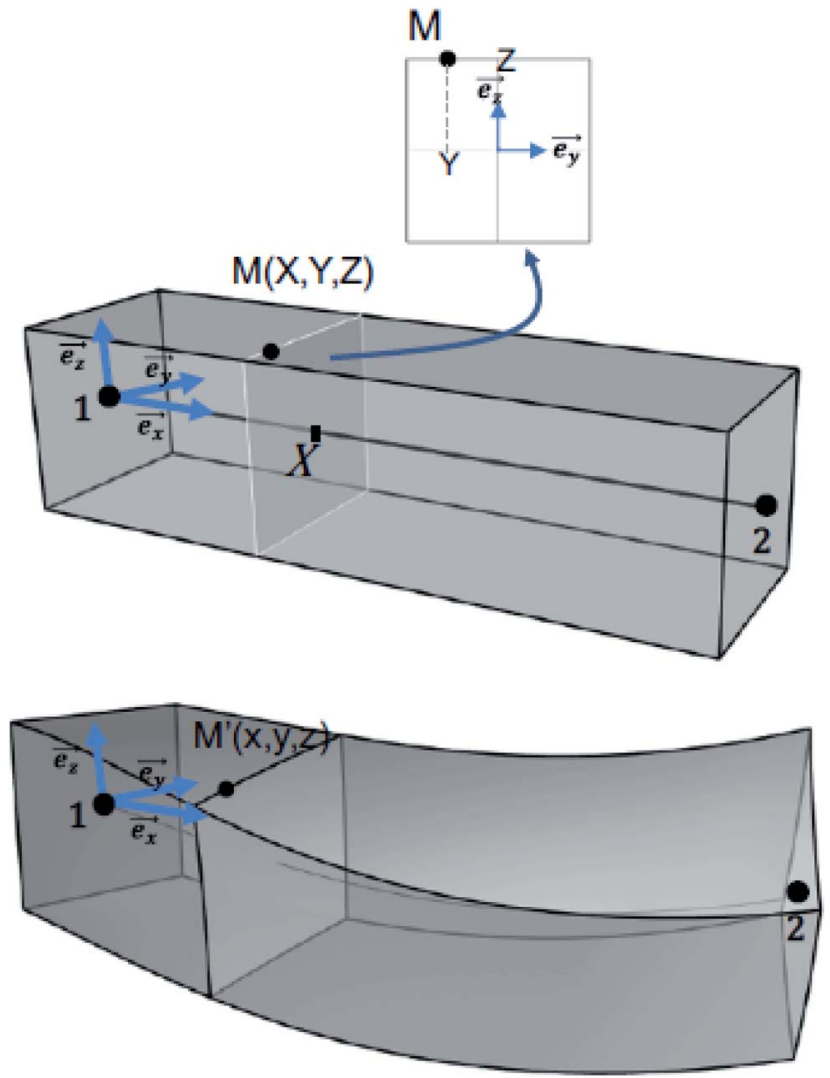

Fig. 1 The beam was mapped along the positive $X$-axis and its centre line was extended from node 1 to node 2 (reprinted with permission from ref. 60. Copyright 2019 Elsevier).

the weights for the beams, $\omega_{i}=\frac{1}{N}$, where $N$ was used for the number of beams. As per the weighted deformation map, each vertex was shifted to study the deformation and thereafter, each voxel was updated for its new position. The validation of the modeling scheme (with finite element method (FEM) based simulations) was done using two different cases, such as (a) using a cantilever beam $(50 \mathrm{~mm} \times 10 \mathrm{~mm} \times 10 \mathrm{~mm})$ having material properties of structural steel (e.g., $E=20 \mathrm{GPa}, G=$ $7.69 \mathrm{GPa}$ being loaded with an $800 \mathrm{~N}$ force at its free end), and (b) using a squared plate $(30 \mathrm{~mm} \times 30 \mathrm{~mm} \times 2 \mathrm{~mm})$ whose sides were fixed and having material properties of homogeneous MD (e.g., $E=1000 \mathrm{GPa}, G=385 \mathrm{MPa}$ being loaded with 16.5 MPa pressure at its center). ${ }^{60}$

The maximum displacements of the above two cases were obtained in the range of the results of the finite element analysis (FEA) shown in Table $1 .^{60}$

The shape memory, ${ }^{76-79}$ piezoelectric, and thermoelectric or photovoltaic effects were incorporated into a smart, bio-inspired, hydrogel-based 4D material (having shape-changing features) for its improved stimuli responses. ${ }^{80-83}$ The smart, bio-inspired, 4D materials are discussed in two categories: (a) non-programmable one in which the shape-changing event is predefined (in such cases, the isotropic expansion and contraction are specified during fabrication) and limited to dimension change, and (b) programmable one in which the shape-changing event is the
Table 1 Values of maximum displacement $(\mathrm{mm})$ (reprinted with permission from ref. 60. Copyright 2019 Elsevier)

\begin{tabular}{lll}
\hline & $\begin{array}{l}\text { Voxel-based modeling } \\
\text { and simulation approach }\end{array}$ & FEA \\
\hline Cantilever beam & 1.86 & 2.03 \\
Square plate & 3.7 & 3.6
\end{tabular}

function of the pathway (in such cases, the isotropic expansion and contraction are not specified during fabrication), or they can be programmed to change their shapes. Such thermomechanically programmed, bio-inspired $4 \mathrm{D}$ material was modeled using the property of shape memory material. ${ }^{84,85}$

Sossou et al. (2019) have proposed stiffness equations for such non-programmed smart material by using the effects of the initial force, as shown in eqn (14). ${ }^{60}$

$$
\overline{K u}=\bar{f}_{M}+\bar{f}_{(\mathrm{S})}, \quad \Delta L=L \times g(\alpha, S), \text { and } \varepsilon_{\mathrm{s}}=\frac{\Delta L}{L}=g(\alpha, S)
$$

In eqn (14), the symbol ' $S$ ' was used to represent the stimulus. In the unloading condition, the length of the bar member (considered for the study) was set free to increase its length from $L$ to $L+\Delta L$. The $\Delta L$ was expressed in terms of the stimulus and material properties. The symbol ' $\alpha$ ' was used to represent the vector connecting the properties of the piezoelectric, electrostrictive, magnetostrictive, photostrictive, and hydrogel materials. The strains corresponding to the stimulus was expressed using eqn (14).

The bar was subjected to an axial force, $F$, which in turn was responsible for the stress, $\sigma=\frac{F}{A}$ (where, $A$ was used to represent the cross-sectional area of the bar). Thereafter, the strain, $\varepsilon_{M}=\frac{\sigma}{E}$, was induced. The overall internal force, $F$, was expressed using eqn (15). ${ }^{60}$

$$
\frac{E A}{L}\left(\bar{u}_{x j}-\bar{u}_{x i}\right)=\sigma A+E A g(\alpha, S)=F\left[\begin{array}{llll}
f_{x i} & f_{y i} & f_{x j} & f_{y j}
\end{array}\right]
$$

In eqn (15), ' $\sigma A$ ' and ' $E A g(\alpha, S)$ ' were used to represent the mechanical force and stimulus-induced force, respectively.

The stiffness equation of the member was shown using eqn (16). ${ }^{60}$

$$
\frac{E A}{L}\left[\begin{array}{cccc}
1 & 0 & -1 & 0 \\
0 & 0 & 0 & 0 \\
-1 & 0 & 1 & 0 \\
0 & 0 & 0 & 0
\end{array}\right]\left[\begin{array}{c}
\bar{u}_{x i} \\
\bar{u}_{y i} \\
\bar{u}_{x j} \\
\bar{u}_{x j}
\end{array}\right]=\bar{f}_{M}+\bar{f}_{S}
$$

The stimulus-induced force was expressed using eqn (17). ${ }^{60}$

$$
\bar{f}_{S}=E A g(\alpha, S)\left[\begin{array}{llllllllllll}
-1 & 0 & 0 & 0 & 0 & 0 & 1 & 0 & 0 & 0 & 0 & 0
\end{array}\right]^{\mathrm{T}}
$$

The piezoelectric effect was modeled using the set of eqn (18). ${ }^{86}$ 


$$
\left.\begin{array}{rl}
E_{j} & =d_{i j} E_{i} \\
g(\alpha, S) & =d_{3 j} E_{3}
\end{array}\right\}
$$

In the set of eqn (18), $E_{i}, \varepsilon_{j}$, and $d_{i j}$ were used to express the electric field (having directions, $i \in[1,3]$ ), the strain (in the direction $j \in[1,6]$ ), and the piezoelectric strain coefficients, respectively. The symbols ' $d_{33}$ ' (along the electric field's polling direction) and ' $d_{31}$ ' (perpendicular to the electric field) were the most used piezoelectric coefficients, and these were thus characterized by the electric field direction $(x, y$ or $z)$. The function, $g(\alpha, S)$, was used to express the electric field direction of the material. Sossou et al. (2019) achieved maximum strain in this situation. ${ }^{60}$

The effect of electrostrictive material was introduced with the incorporation of dielectric materials. ${ }^{83}$ The second order mathematical expression was positively used to describe the final deformation (proportional to the square of the electric field). The static dielectric constant, $\in$, of the material (as the polarization vector, $P=\left[\begin{array}{lll}P_{1} & P_{2} & P_{3}\end{array}\right]$ was the function of the electric field in such a way that $P_{1}=\in E_{1}$, where $P_{1}$ is the only polarization direction of the electric field and thus, $P_{2}$ and $P_{3}$ were substituted with zeros in the relationship of induced deformations and polarization) was expressed using a set of the set of eqn (19). ${ }^{60,87}$

$$
\begin{aligned}
& \epsilon_{1}=Q_{11} \in{ }^{2} E_{1}{ }^{2}=M_{11} E_{1}{ }^{2} \\
& \epsilon_{2}=Q_{12} \in{ }^{2} E_{1}{ }^{2}=M_{12} E_{1}{ }^{2} \text { in general } g(\alpha, S)=M_{1 j} E_{1}{ }^{2} \\
& \epsilon_{3}=Q_{13} \in{ }^{2} E_{1}{ }^{2}=M_{12} E_{1}{ }^{2}
\end{aligned}
$$

The function $g(\alpha, S)$ was expressed as $g(\alpha, S)=M_{1 j} E_{1}{ }^{2}$, where $E_{1}$ was the direction of the applied electric field, and the symbols ' $M_{11}$ ', and ' $M_{12}$ ' were used as electrostrictive material properties along the direction of the applied electric field and in the direction perpendicular to the applied electric field, i.e., the transverse direction, respectively.

The effect of electrostrictive material was introduced with the incorporation of magnetostrictive properties, ${ }^{66}$ such as $\lambda_{s}$ (the strain of magnetostrictive material at its magnetization saturation) and $M_{\mathrm{s}}$ (the saturation stage of magnetic field), and was shown using the linear eqn (20). ${ }^{60}$

$$
g(\alpha, S)=\lambda=\left\{\begin{array}{l}
\frac{\lambda_{\mathrm{s}}}{M_{\mathrm{s}}}|M|,|M|<M_{\mathrm{s}} \\
\lambda_{\mathrm{s}},|M| \geq M_{\mathrm{s}}
\end{array}\right.
$$

The effect of photostrictive (light sensitive) materials ${ }^{\mathbf{8 8 , 8 9}}$ was introduced after restraining the need of photostriction to the intensity of light (as shown in eqn $(21)^{90}$ ).

$$
\frac{\Delta L}{L}=k I
$$

In eqn (21), the symbols ' $T$ ' and ' $k$ ' were used to represent the intensity of light and material intensity, respectively.

The hydrogels were used as a sponge to repel or absorb water under wet conditions. The volumetric swelling ratio (VSR) was calculated using the eqn $(22)^{81}$ ).

$$
v_{\mathrm{s}}=\frac{V_{0}}{V_{\mathrm{s}}} g(\alpha, S)=\left[v_{\mathrm{s}}(T)\right]^{\frac{-1}{3}}-1
$$

In eqn (22), the symbols ' $V_{0}$ ' and ' $V_{\mathrm{s}}$ ' were used to represent the volume of material in the dry state and in the swollen state, respectively. The value of ' $v_{\mathrm{s}}$ ' was in between 0 and 1 . The function $g(\alpha, S)$ was expressed as $g(\alpha, S)=\left[v_{\mathrm{s}}(T)\right]^{\frac{-1}{3}}-1$ (where $T$ was the temperature, and $v_{\mathrm{s}}(T)$ was used to represent the dependency of VSR on temperature) to describe the internal force (on the beam made of hydrogel material) due to the temperature-driven volume change.

In the model discussed above, stimuli such as the electric field, light, heat, magnetic field and voltage were sensed by voxels and beams. The voxel-based modeling and simulation method was validated with few existing cases of heat-sensitive hydrogels such as the $4 \mathrm{D}$ printed smart hydrogel valve and actuators (theoretical) ${ }^{81,91}$ of particular material distributions (MDs). The results simulated were in good agreement with experimental, as well as finite element analysis (FEA) results, ${ }^{60}$ as shown in Fig. 2. The shrinking of the hydrogel material was observed with its bending response (Fig. 2). The active and passive hydrogels were shown using red and blue colors, respectively, as shown in Fig. 2.

\subsection{Modifications in the voxel-based modeling framework, using exponential expressions to encode the time-dependent behavior}

The shape-changing features in the $4 \mathrm{DP}$ materials are encoded with different forms of anisotropy (for relative expansion between active and passive substances) induced after fabricating with various heterogeneous materials. The properties of

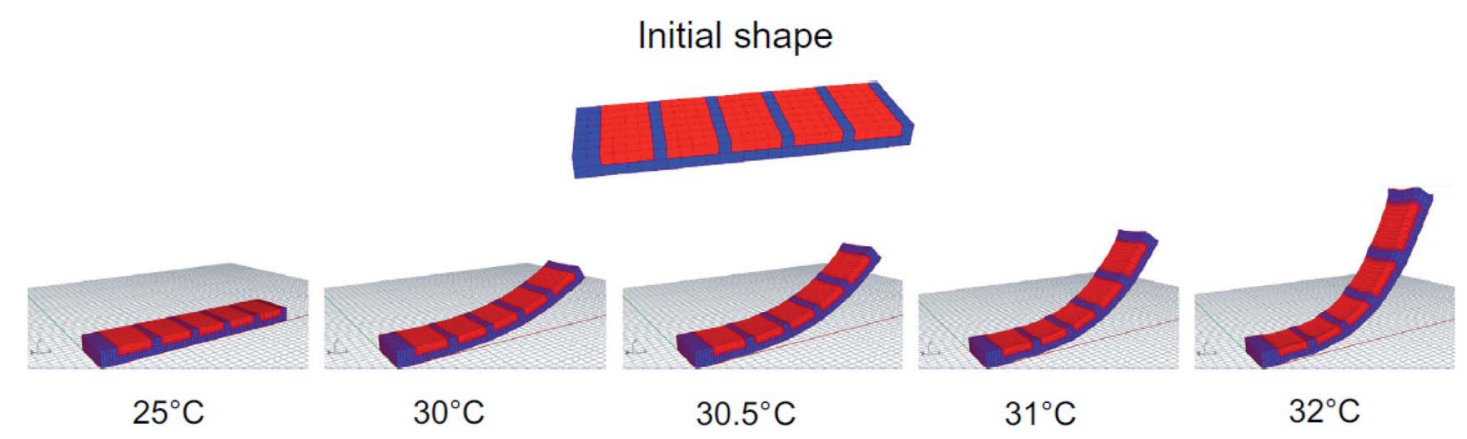

Fig. 2 Illustration of voxel-based modeling and simulation approach for smart hydrogel actuator (the left end of the hydrogel was fixed during the simulation) (Reprinted with permission from ref. 60. Copyright 2019 Elsevier). 
the piezoelectric, electrostrictive, magnetostrictive, photostrictive, and hydrogel materials are programmed in such thermo-mechanically trained bio-inspired materials, so that they can recover their permanent shapes. The shape-changing properties of the bio-inspired 4DP materials are governed through the physics of thermal expansion and contraction, and organic advancement.

The thermal expansion and contraction of 4DP materials (having the properties of piezoelectric, electrostrictive, magnetostrictive, and photostrictive materials) can be expressed through the strain eqn (23). ${ }^{2}$

$$
\varepsilon_{\text {thermal }}=\alpha \Delta T
$$

In eqn (23), the symbols ' $\alpha$ ' and ' $\Delta T$ ' were used to represent the coefficient of thermal expansion and the change in temperature, respectively. The 4DP materials were modeled using the initial force function, $\bar{f}_{(\mathrm{s})}$, which was in terms of the stimulus, $S$. Further, the change in the length of bar, $\Delta L$, was expressed using eqn (14). The function $g(\alpha, S)$ (used in eqn (18)-(20)) could be modified for the thermal stimulus that evokes the responsive $4 \mathrm{DP}$ materials. A temperature, $T_{2}$, should be added as the thermal stimulus in the $4 \mathrm{DP}$ material with an initial temperature $T_{1}$ to encode its behavior with time, $t,,^{92,93}$ as shown in eqn (24).

$$
\begin{aligned}
& \varepsilon_{\text {thermal expansion/contraction or mass diffusion or organic growth }}(t) \\
& \quad=C\left[1-\exp \left(-\frac{t}{\tau}\right)\right]
\end{aligned}
$$

In eqn (24), the symbols ' $C$ ' and ' $\tau$ ' are constants. The time constant, $\tau$, depends on the mechanical properties (such as thermal capacity and density), attributes of the solid (such as volume), and thermal resistivity. The time-based eqn (24) is a modified form of the temperature-based eqn (23), which can take care of stimuli, such as piezoelectric, electrostrictive, magnetostrictive, and photostrictive mechanisms. The materials that are responsive to electrothermal stimuli will expand after the passage of current due to the ohmic heating, and thus contract upon cooling. However, in the few cases where the materials contract upon heating, the coefficient of thermal expansion, $\alpha$, is negative and thus eqn (24) would remain unchanged.

The property of hydrogels, such as the ability to absorb or desorb water, has been characterized using VSR in eqn (22). The function of $g(\alpha, S)$ can be modified for the 4DP material (where the mass changes due to the sorption of water) in terms of time, $t,{ }^{94-96}$ as shown in eqn (24). The isotropic material will have $\varepsilon_{x}=\varepsilon_{y}=\varepsilon_{z}=\varepsilon=\frac{1}{3} \frac{\Delta V}{V}$, and due to the linear relationship between the mass and volume, eqn (24) fits for the mass diffusion. ${ }^{94}$ The time-based eqn (24) will fit for the stimuli, which are responsible for the transportation of matter to cause the expansion in the 4DP structures.

Our study also suggests the importance of incorporating the effect of organic growth over time in the bio-inspired 4DP material to deal with cells, scaffolds, and tissues in the field of bioscience or bioengineering. The organic growth should be modeled as an increase in length or weight, as shown in eqn (24). The constants, $C$ and $\tau$, are the ratio of the change in length to the initial length, and the time-constant (a function of the metabolism of a living organism), respectively. The shape-changing behavior of the 4DP materials (having active and passive layers) is time-dependent and can be expressed in terms of curvature, as expressed using eqn $(25) .^{2}$

$$
\kappa(t)=\frac{1}{\rho}(t)=K_{\mathrm{I}}\left(1-\mathrm{e}^{\frac{-t}{\tau_{\mathrm{I}}}}\right)+K_{\mathrm{II}}\left(1-\mathrm{e}^{\frac{-t}{\tau_{\mathrm{II}}}}\right)
$$

In eqn (25), $t, \kappa(t)$, and $\rho$ are used to express the time, curvature (due to relative expansion), and radius of the curvature, respectively. $K_{\mathrm{I}}$ and $K_{\mathrm{II}}$ are constants that depend on Young's moduli of passive and active layers, as well as the moment of inertia, and the stress at the interface. The time constant, $\tau_{\mathrm{I}}$, depends on the viscosity (at the interface of layers) and Young's moduli. Eqn (25) is used when the 4DP material has changed its shape, i.e., when the stimulus is "active (stimulus on)". If the stimulus is "not active (stimulus off)", then the 4DP material has to restore its initial shape, as shown in eqn (26). ${ }^{2}$

$$
\left(\frac{1}{\rho}\right)_{\text {not active }}=K_{\mathrm{I}} \mathrm{e}^{\frac{-t}{\tau_{\mathrm{I}}}}+K_{\mathrm{II}} \mathrm{e}^{\frac{-t}{\tau_{\mathrm{II}}}}
$$

The graph representing eqn (25) and (26) is shown in Fig. 3. The time-dependent bi-exponential expressions were validated with experimental data available in the literature, and found fit to predict the nonlinear behavior of the $4 \mathrm{D}$ printed materials.

\subsection{D printing of the composite hydrogel}

Fabrication of the plant-like materials, such as flowers, that could change shapes when being immersed in water or while responding to light or touch, was done using the $4 \mathrm{D}$ printing

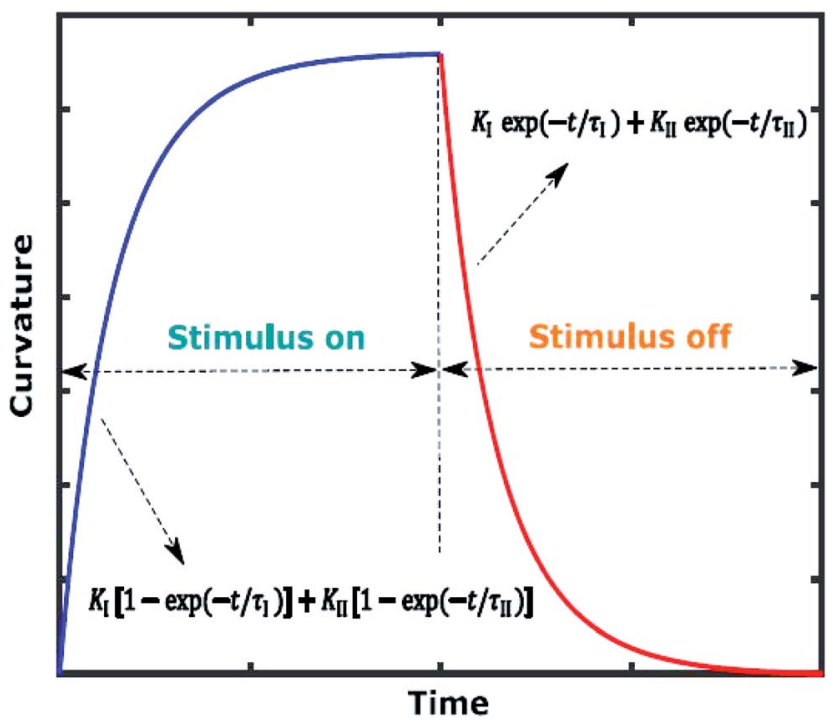

Fig. 3 The behavior of the 4DP material as a function of time (reprinted with permission from ref. 2. Copyright 2019 Cornell University). 

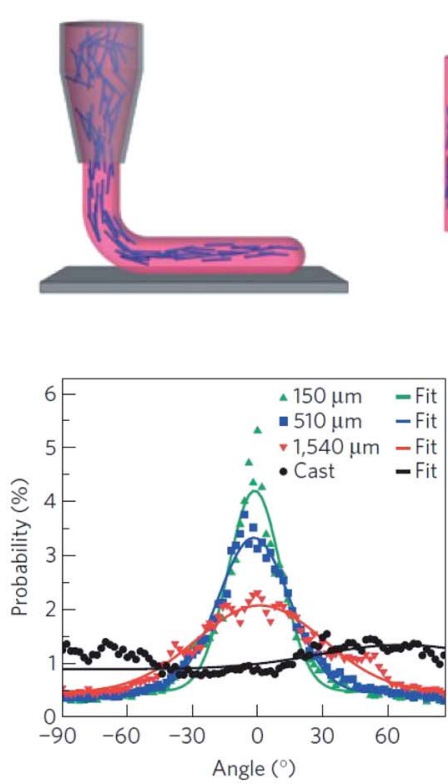

(b)

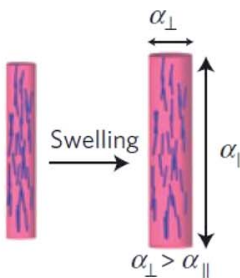

$E_{\|}>E_{\perp}$

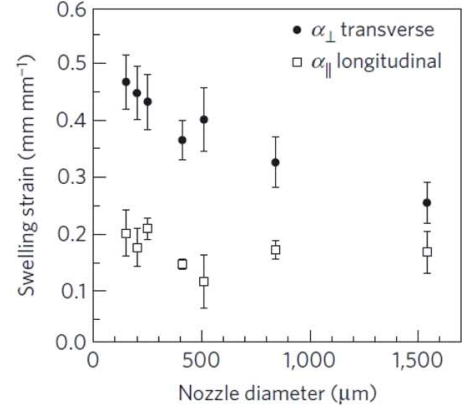

(c)

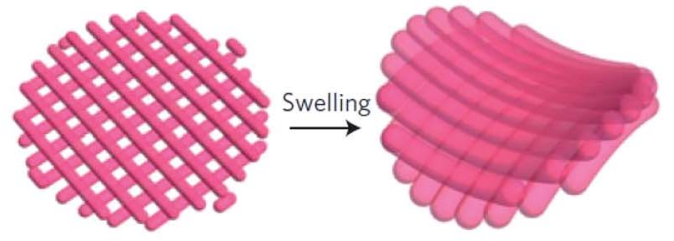

(a)

Fig. 4 The local anisotropy was programmed through bio-inspired 4D printing (reprinted with permission from ref. 54. Copyright 2016 Springer Nature). Single-step alignment of cellulose fibers during 4D printing. The (a) Anisotropic stiffness $(E)$ and swelling strain $(\alpha)$ were influenced during direct ink writing, and an illustration of the shear-induced alignment of cellulose fibrils was shown in the figure. (b) A peak was observed at $0^{\circ}$, which had quantified directionality in case of unidirectional samples. However, the isotropic samples did not show any peak. (c) The transverse and longitudinal swelling behavior was dependent on the nozzle diameter during the 4D printing of the hydrogel composites. (d) Data on the swelling strains were plotted for the nozzle diameter of $510 \mu \mathrm{m}$. *Note: the data were in terms of the mean \pm standard deviation, $n=6$.

technique. $^{54}$ It was done after using a suitable threedimensional mathematical model that involved anisotropic swelling to have control over the embedding of the complicated surface. Gladman et al. (2016) have successfully printed the 4D composite hydrogel that has been encrypted with a decentralized, anisotropic enlargement feature under the restrained alignment of cellulose fibers along the four-dimensional pathways. $^{54}$ The materials, incorporated with shape memory elements and swellable hydrogels, were believed to respond to external stimuli, ${ }^{6497-100}$ but none of these were effective in terms of changing their shapes upon stimulation. To overcome the limitations of the existing materials, a $4 \mathrm{D}$ printed biomimetic hydrogel composite has been developed through the programming of bilayer architectures patterned in space and time. ${ }^{54}$ The object printed so far has the capability to change the shapes on being immersed in water, as these were encoded with localized swelling anisotropy.

The mechanics of anisotropic plates and shells is required as a mathematical model to study the curvature in the bilayer structures (precise control is required over the curvature, and that too is possible after harnessing the anisotropic swelling). ${ }^{101,102}$ The classical approach of the Timoshenko model was combined for the thermal expansion in the bilayer, along with a metric-driven approach (to consider anisotropic swelling while in the complex surface). In a bilayer system, the top and bottom layers were supposed to remain in contact along the entire mid-plane. In such system, the differential swelling between the two layers has involved a curvature. The displacements (integrated from the swelling and curvature strain tensors) and tractions along the mid-plane were then identical. All these conditions were involved under a threedimensional model for the $4 \mathrm{D}$ printed structures. ${ }^{54}$

Here, the bottom layer was printed in the ' $e_{x}$ ' direction, and the top layer was further rotated by ' $\theta$ ' degrees in the anticlockwise direction. The curvature, thus formed, was dependent upon the elastic moduli and swelling ratios. The ratio of the thicknesses of layers $(m)$ and overall bilayer thickness $(h)$ was shown using eqn (27). ${ }^{54}$

$$
m=a_{\text {bottom }} / a_{\text {top }}
$$

The total thickness of the bilayer was shown in eqn (28). ${ }^{54}$

$$
h=a_{\mathrm{top}}+a_{\mathrm{bottom}}
$$

The Gaussian $(K)$ and mean $(H)$ curvatures were represented using the set of eqn (29). ${ }^{54}$

$$
\left.\begin{array}{l}
K=-c_{4} \frac{\left(\alpha_{\|}-\alpha_{\perp}\right)^{2}}{h^{2}} \frac{\sin ^{2}(\theta)}{c_{5}-c_{6} \cos (2 \theta)+m^{4} \cos (4 \theta)} \\
H=c_{1} \frac{\alpha_{\|}-\alpha_{\perp}}{h} \frac{\sin ^{2}(\theta)}{c_{2}-c_{3} \cos (2 \theta)+m^{4} \cos (4 \theta)}
\end{array}\right\}
$$

In the above eqn (29), the symbol ' $c_{i}$ ' was used for the elastic constants, the limit $\theta \rightarrow 0^{\circ}$ was used for the Timoshenko equation, and the condition $\theta=90^{\circ}$ was used to achieve the saddle-shaped structure. 


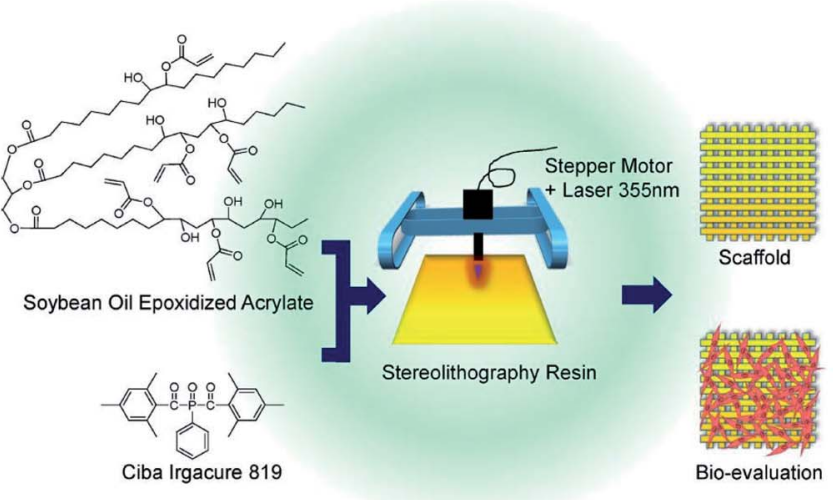

(a)

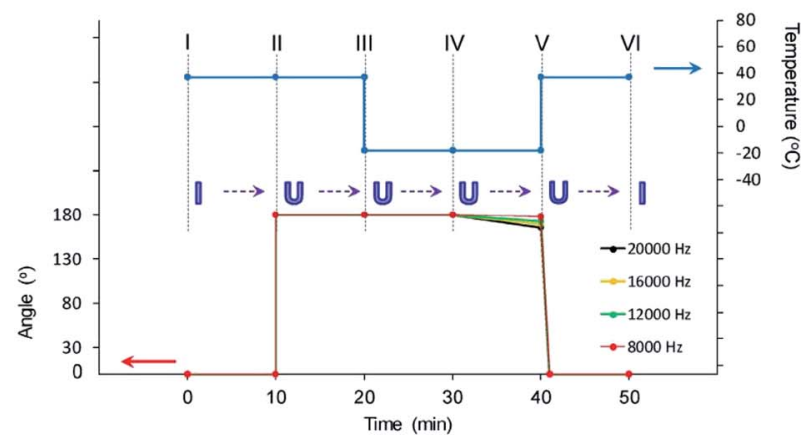

(c)
Infill $20 \% \quad$ Infill $30 \% \quad$ Infill $40 \% \quad$ Infill $50 \%$
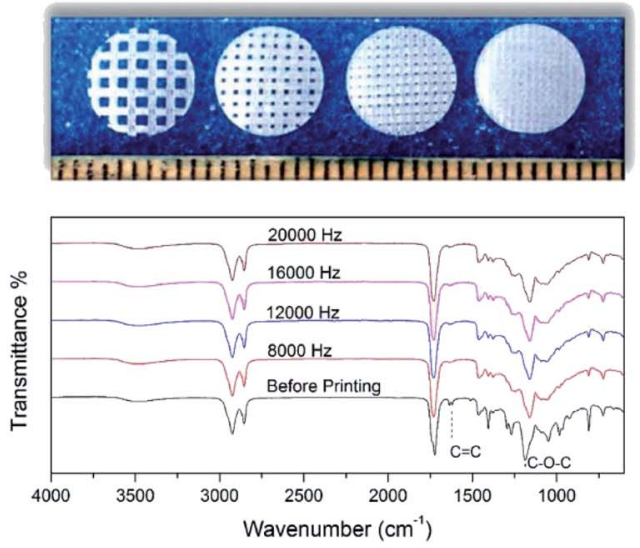

(b)

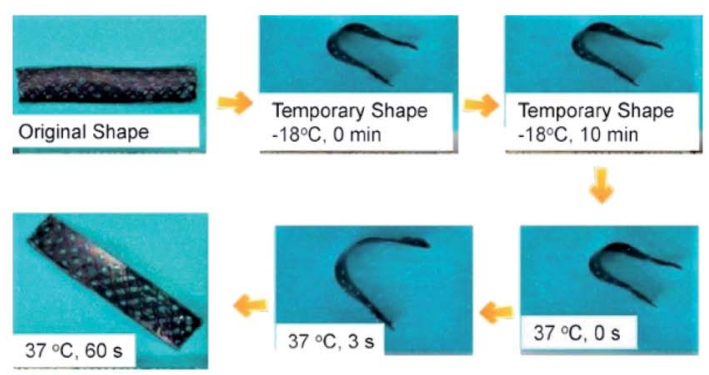

(d)

Fig. 5 Printing of biocompatible scaffolds (reprinted with permission from ref. 30. Copyright 2019 Springer Nature Limited). (a) The schematic representation of 4D printed biocompatible scaffolds with renewable soybean oil using the multi-dimensional stereolithography method. (b) The 4D printed scaffolds (laser frequency and the speed of printing were set to $12000 \mathrm{~Hz}$ and $10 \mathrm{~mm} \mathrm{~s}^{-1}$, respectively) with various infill densities. The polymerization of the soybean oil epoxidized acrylate was confirmed through FTIR analysis. (c) The shape-changing cycle of the printed scaffolds at different laser frequencies. (d) The stages of the shape-changing biocompatible scaffold printed with a speed of $10 \mathrm{~mm} \mathrm{~s}^{-1}$ at a laser frequency of $12000 \mathrm{~Hz}$. *Note: the data were in terms of the mean \pm standard deviation, $n=6$.

The efficiency of the $4 \mathrm{D}$ printing method was based on the ability to deterministically specify the elastic and swelling anisotropies using the local control of the orientation of the cellulose fibrils of the hydrogel composite (as shown in Fig. 4(a)). In Fig. 4(a), the fibrils were shown to undergo shearinduced alignment due to the flow of ink through the deposition nozzle. Thus, the filaments (having anisotropic stiffness) were printed. Further, the swelling behavior in the longitudinal and transverse directions was studied in Fig. 4(a). The considerable shear-induced alignment was observed in the $4 \mathrm{D}$ printed samples. The directionality peak of the Fourier analysis has established the relative alignment in the printed specimens (as shown in Fig. 4(b)). The existence of a four-fold difference in the longitudinal and transverse strains in the $4 \mathrm{D}$ printed objects was marked through $\alpha_{\|} \sim 10 \%$ and $\alpha_{\perp} \sim 40 \%$, respectively (as shown in Fig. 4(c) and (d)). In the same way, the footprint of the anisotropy was reported in the elastic moduli (the longitudinal and transverse values were $E_{\|} \sim 40 \mathrm{kPa}$ and $E_{\perp} \sim 20 \mathrm{kPa}$, respectively). The authors have reported that the shear-induced alignment and the anisotropic swelling were all dependent upon the nozzle diameter and printing speed. ${ }^{54}$
2.5 4D printing of the smart biomimetic scaffolds to support the growth of human mesenchymal stem cells (hMSCs), and applications of the biomimetic dual shape-changing tube in human engineering as a bioimplant (such as the vascular graft)

The renewable and economical polymers of plant oils could serve as better alternatives to the limited and depleting conventional petroleum-based biopolymers, due to their (plant oils polymers) improved biocompatible features apart from their wide availability in nature. The ink for the printing of the biocompatible scaffolds was prepared by mixing $100 \mathrm{~g}$ of soybean oil epoxidized acrylate with $10 \mathrm{~mL}$ acetone before adding $1.26 \mathrm{~g}$ bis(2,4,6trimethylbenzoyl)-phenylphosphine oxide into the mixture. ${ }^{30}$ The solution was then kept overnight into the properly washed and dry-cleaned chamber to separate acetone.

The stereolithography method is found to be the most efficient method to print biomaterials (such as $4 \mathrm{D}$ scaffolds) using plant oils (the renewable stocks as feeds for biopolymers) through computer-aided interfaces. ${ }^{103-107}$ The shape-changing $4 \mathrm{D}$ materials are printed after reinforcing the shape memory polymer fibers in the elastomer matrix. ${ }^{108}$ The shape-changing 
feature of such bio-inspired materials is a function of time, as discussed above, and thus has potential application as biocompatible scaffolds. ${ }^{109}$ The liquid form of the soybean oil epoxidized acrylate renewable resin ${ }^{110}$ was used to print biocompatible scaffolds ${ }^{111}$ using the multi-dimensional stereolithography method..$^{\mathbf{3 0 , 1 1 2}}$

The soybean oil ${ }^{113-115}$ epoxidized acrylate resin was solidified using multi-dimensional laser printing for the smart biocompatible scaffolds (as shown in Fig. 5(a) and (b)) to support the growth of human bone marrow mesenchymal stem cells (hMSCs). ${ }^{30}$ The scaffolds were printed using a laser frequency of $12000 \mathrm{~Hz}$ at a speed of $10 \mathrm{~mm} \mathrm{~s}^{-1}$. It was observed that, with the increase in the infill density from 20 to $50 \%$, the pores in the printed scaffolds were reduced significantly (Fig. 5(b)). The Fourier transform infrared spectroscopy spectrometer was used to approve the polymerization of the soybean oil epoxidized acrylate renewable resin. With the increase in the signals from
1620 to $1640 \mathrm{~cm}^{-1}$, the double bonds between the carbon atoms were stretched in the acrylic acid. These signals disappeared to confirm the polymerization of the bonds. Also, there was a shift in the signals (as shown in Fig. 5(b)) for the wavelength, changing from 1186 to $1177 \mathrm{~cm}^{-1}$, which further confirmed the presence of $\mathrm{C}-\mathrm{O}-\mathrm{C}$ in the ester group. The shape memory effects of the scaffolds were plotted in relation with time and temperature (as shown in Fig. 5(c)). The entire cycle of the shapechanging events was captured (I-VI). Initially, the sample scaffold had a U-shape at $37^{\circ} \mathrm{C}$, and it was preserved in this stage at the same temperature for the next $10 \mathrm{~min}$ (II-III). Then, the temperature was reduced to $-18{ }^{\circ} \mathrm{C}$ and the scaffold was preserved in the same stage for the next $10 \mathrm{~min}$ (III-IV). Finally, the external support was removed to set the scaffold to retain its initial shape (IV-V). The scaffolds printed at laser frequencies of $8000 \mathrm{~Hz}$ and $20000 \mathrm{~Hz}$ have the shape-changing capabilities (shape fixity) of $99 \%$ and $92 \%$, respectively. The temporary

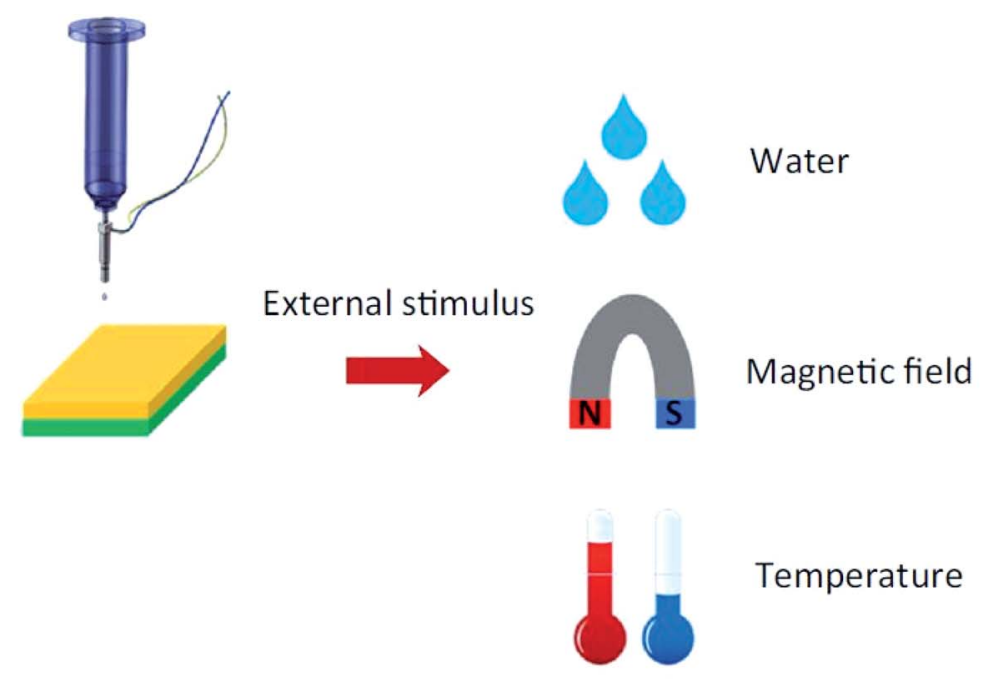

(a)

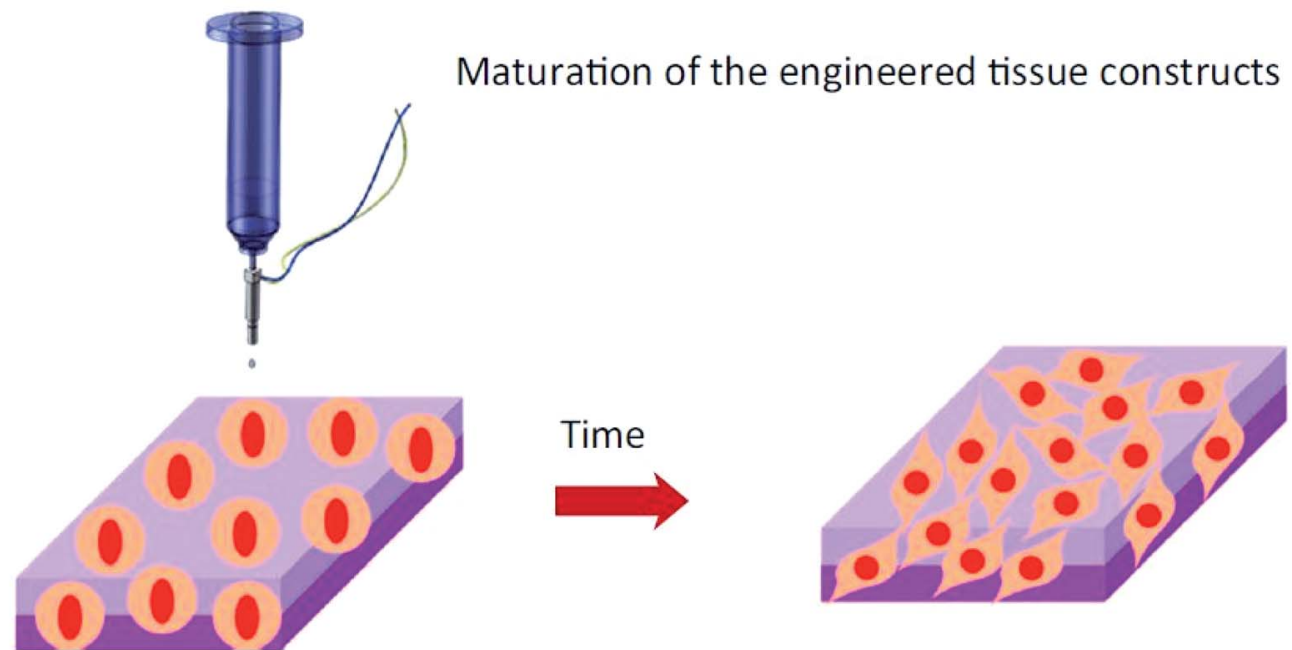

(b)

Fig. 6 Schematic representation of the concepts of the 4D printed materials (reprinted with permission from ref. 116. Copyright 2016 Elsevier Limited). (a) The stimulus-responsive 4D printed smart material. (b) The time-dependent 4D printed assembled tissue design. 
shape of the biomedical scaffolds was achieved at $-18{ }^{\circ} \mathrm{C}$. However, the permanent shape was restored at $37^{\circ} \mathrm{C}$ (as shown in Fig. 5(d)).

The cytotoxicity test of biocompatible scaffolds was performed (to prove its cytocompatibility), and found to have significant adhesion and proliferation of hMSCs. The renewable liquid resin has a higher adhesion and proliferation of hMSCs compared to the established polyethylene glycol diacrylate (PEGDA). The scaffold (with 70\% infill density), printed using a laser frequency of $12000 \mathrm{~Hz}$ at a speed of $10 \mathrm{~mm} \mathrm{~s}^{-1}$, was observed to have a considerably higher attachment potential to hMSCs than PEGDA. The test was further extended with the scaffold having $20 \%$ infill density. It concluded with the growth tendency of hMSCs along the creased pattern on the facet of the scaffold's plane, contrary to the PEGDA that was lacking the ability to hold bio active features, as only a few types of cells functionally stick and develop on its uppermost layer. It was found that the wettability of any set surface by the soy scaffold (water contact angle in case of the soy resins) was almost the same as that of the outstanding cytocompatible polycaprolactone (PCL). ${ }^{30}$ Besides this, the enhanced biocompatibility of the soybean oil epoxidized acrylate renewable resin was also due to the presence of the cyto-friendly hydroxyl and ester chemical groups.

The essential parameters, such as the shape fixity $\left(S_{\mathrm{f}}\right)$ and shape recovery $\left(S_{\mathrm{r}}\right)$, for the $4 \mathrm{D}$ printed scaffolds have been calculated using the set of eqn (30).

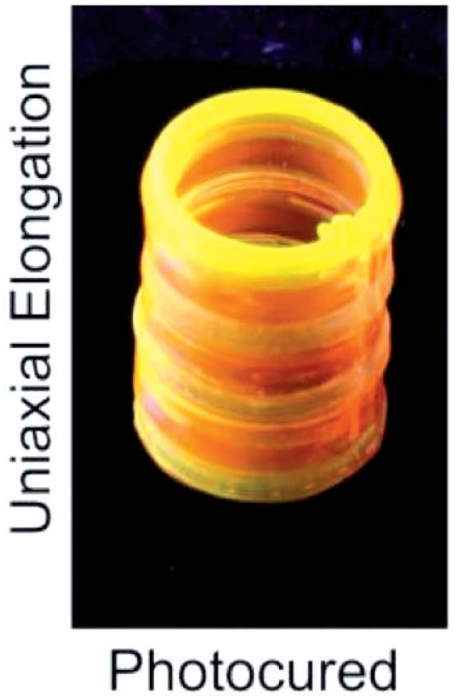

(a)

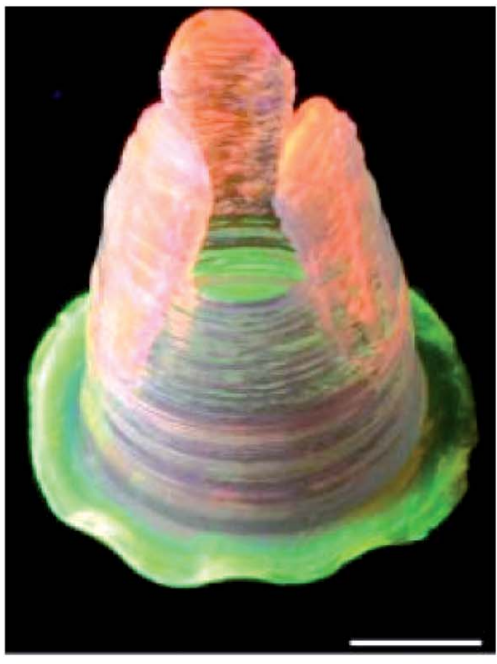

Photocured

(d)

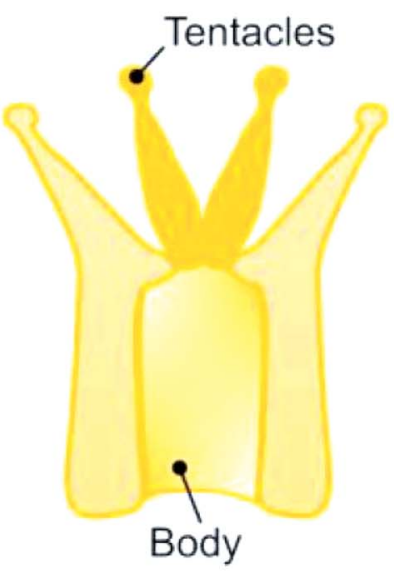

Coral Polyp

(b)

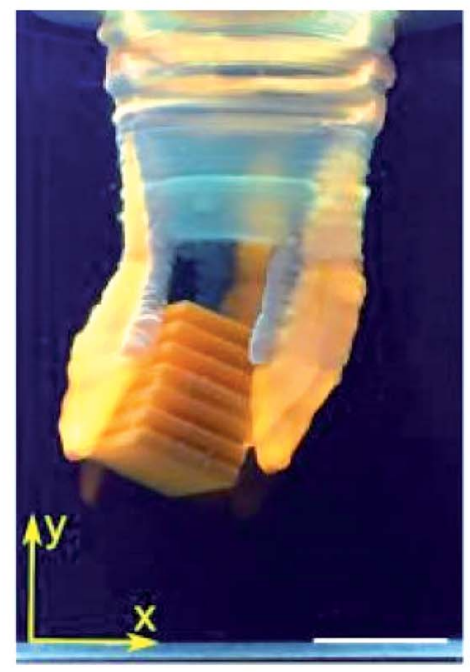

Lifted at $25^{\circ} \mathrm{C}$ in Water

(e)

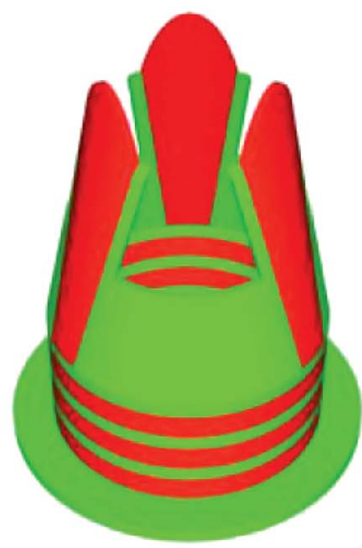

Dual-function Tube

(c)

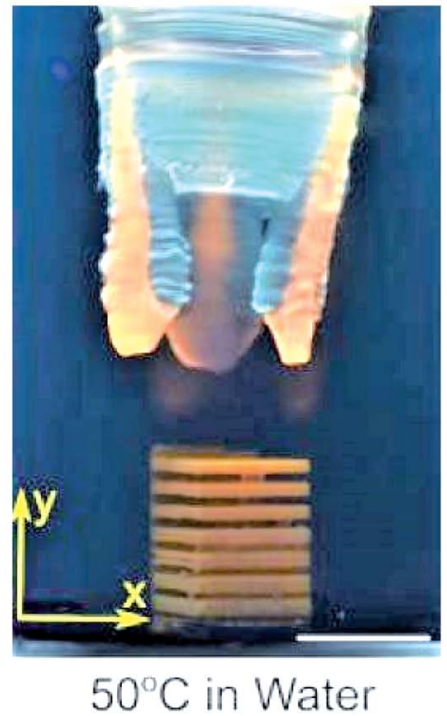

(f)

Fig. 7 The biomimetic 4D printed dual-gel tube (reprinted with permission from ref. 117. Copyright 2019 American Chemical Society). (a) The uniaxial expansion of a dual shape-changing tube. (b) The bodily structure of a coral polyp. (c) The schematic representation of a dual-gel tube. (d) The 4D printed and photocured tube with the fingers. (e) The gripping of (and thereafter lifting up) the cube at $25^{\circ} \mathrm{C}$ due to the uniaxial expansion of the tube when suspended in water. (f) On heating at $50{ }^{\circ} \mathrm{C}$, the fingers were opened up to release the cube. 


$$
\left.\begin{array}{l}
S_{\mathrm{f}}=\frac{\text { fixed increament }\left(\theta_{\text {fixed }}\right)}{\pi} \times 100 \% \\
S_{\mathrm{r}}=\frac{\text { fixed increament }\left(\theta_{\text {fixed }}\right)-\text { final increament }\left(\theta_{\text {final }}\right)}{\text { fixed increament }\left(\theta_{\text {fixed }}\right)} \times 100 \%
\end{array}\right\}
$$

There are basically two types of the $4 \mathrm{D}$ bioprinted smart materials: (a) the shape-changing stimulus-responsive smart materials (Fig. 6(a)) due to their fundamental characteristics, and (b) the time-dependent assembled tissue design (Fig. 6(b)), which generally grows with time after bioprinting. Examples of such stimulus-responsive smart materials include the self- folding structures, and the time-dependent materials include the microtissues (printed using bioinks, such as the cell-laden microfluidic microgels for tissue regeneration), which mature or grow as time passes. ${ }^{116}$

The goals of expansions in particular and radial directions (Fig. 7(a)), as well as curving and grasping, were achieved by utilizing the biomimetic concepts of the symmetrically arranged high and low swelling gels in a dual shape-changing tube (initially inspired by the coral polyp (Fig. 7(b)), an aquatic living creature having such cylindrical shape and was supposed to capture prey using its tentacles). The thermally responsive tube (Fig. 7(c) and (d)) was designed to expand and grasp an object such as a cube, as per the need. ${ }^{117}$ The event was

Table 2 Applications of the 4D printed materials in the field of medical sciences

Applications in medical

sciences

Details

$4 \mathrm{D}$ printed stents

In the $4 \mathrm{D}$ printed stents, length is a function of time. These smart materials can change their lengths with time, and thus are having applications in plastering broken parts of patients

4D printed organs

Potential application in fulfilling the demands of organ shortages; the patient's cells were used while printing his/her organs

4D printed smart multimaterials 4D printed implants (make breathing easier)

$4 \mathrm{D}$ printed implants (tissue engineering applications)

$4 \mathrm{D}$ printed hearts, kidneys, and levers

4D printed skin graft

Potential application in printing the same color skin graft as that of the patient for treatment after burn injuries

$4 \mathrm{D}$ printed smart medical equipments

4D printed CT scanners

Potential application in customizable medical implants

These smart materials are capable of changing their shapes over time, and thus make breathing easier for the babies (who are growing with time)

Potential application in tissue engineering as shape-changing implants

Potential application in printing flexible (that can grow with time) human body parts such as heart, kidneys, and lever (with genetic match) using smart materials

Potential application in producing functional medical equipment, as per the requirement of surgeries

Potential application in surgical planning and improving clinical outcomes for patients
Materials and their printing methods

References

The biomedical devices based on methacrylated polycaprolactone (PCL) shape memory polymers having a molecular weight of $10,000 \mathrm{~g} \mathrm{~mol}^{-1}$ for endoluminal procedures, printed using the UV-LED stereolithography printer

The fully functional human organs could be printed using cell-containing inks. The biocompatible inks were loaded into the syringes of the cartridges in the polyjet printer and the stereolithographic printer

The active hinges based on SMPs, printed using the multimaterials ink jet printer

The splints and scaffolds based on

118

polycaprolactone (safe, as likely to go into the body as time passes) and shape memory polyurethane, respectively, were printed using the conventional 3D printers. The scaffolds' 3D printer was featured with the time-supervised shape morph of the thermosensitive SMPs

The fanciful ball having an airy structure $(1 \mathrm{~mm}$ in diameter), based on a plain hydrogel and alkaline phosphatase, and printed using the digital light processing technique. The cartilage is based on collagen (containing bone morphogenic proteins), and printed using the in-house extrusion-based multi-head printer

The heart based on translucent material, printed using the hybrid printer (having combined features of computed tomography and 3D

echocardiography). The scaffolds such as cells, collagens, etc., could be used to print the skin constructs by dynamic optical projection stereolithography technique The semi-interpenetrating polymer network elastomer (having shape memory and self-healing potential) based on urethane diacrylate and linear semicrystalline PCL, printed using the UV-assisted direct-ink-write printer

The SMPs based on acrylates such as polyurethane, epoxy and isobornyl, along with the radical photoinitiator (such as PI 184), printed using the SL200 stereolithography printer

The photopolymer based on plastic, printed using the hybrid printer (having the combined features of computed tomography and 3D echocardiography)
119 and

121 and 122 123 and 124

125 and 126

127 and 128 
accomplished at $25{ }^{\circ} \mathrm{C}$ after the successive uniaxial expansion of the shape-changing tube, further curving its fingers to grab the cube (Fig. 7(e)) without harming its surface, and finally releasing the cube while opening up its fingers (Fig. 7(f)) when heated to $50{ }^{\circ} \mathrm{C}$.

The $4 \mathrm{D}$ printed dual-gel tube was fabricated using a thermally (a) responsive (active) gel, such as poly $\mathrm{N}$-isopropylacrylamide (pNIPAM), and a (b) non-responsive (passive) gel, such as polyacrylamide (pAAM). The physical behavior of the bio-inspired tube was achieved after the controlled swelling of the gels, and was further validated well using the results of the finite element analyses. The active ink was prepared by dissolving LAPONITE® $(6.77 \%$ by weight $)$ into the deionized (DI) water ( $84.6 \%$ by weight). The dye of a $0.12 \mathrm{mg} \mathrm{mL}^{-1}$ solution of methacryloxyethyl thiocarbamoyl rhodamine B (10 vol\%) was added to the LAPONITE® solution to visualize changes in the biomimetic substances. The monomer of $\mathrm{N}$-isopropylacrylamide $(8.46 \%$ by weight $)$ was added to the LAPONITE $®$ solution, along with Irgacure $2959(0.17 \%$ by weight), to catalyze the event in the presence of light to improve the solubility. The passive ink was prepared by dissolving LAPONITE® $(6.64 \%$ by weight) into the deionized (DI) water (82.97\% by weight). The dye of a $0.3 \mathrm{mg} \mathrm{mL}{ }^{-1}$ solution of fluorescein $o$-methacrylate $(0.0025 \%$ by weight $)$ was added to the LAPONITE® solution, along with $2 \mathrm{mg} \mathrm{mL}{ }^{-1}$ of $\mathrm{NaOH}$ $(0.017 \%$ by weight) to visualize changes in the biomimetic substances. The monomer of acrylamide ( $7.88 \%$ by weight), and $N, N^{\prime}$-methylenebisacrylamide $(1.66 \%$ by weight $)$ as a crosslinker were added to the LAPONITE® solution, along with Irgacure 2959 ( $0.83 \%$ by weight) to catalyze the event in the presence of light to improve the solubility.

The inks were loaded into the syringes of the cartridges in the Direct Ink Writing Printer, and further kept at room temperature overnight. As per the STL files, the tubes were fabricated and photocured after UV exposure. The stress- strain curves were plotted for the active and passive hydrogel panels to evaluate their Young's moduli. The elongations in the active and passive segments were measured through FEA simulations, and found to be in agreement with the experimental data sets.

\section{Future applications}

The study of biomimetic 4D printing opens a new avenue for creating objects that are responsive to environmental stimuli, such as humidity, touch, or even light, through their shapemorphing properties. These materials have high-end applications in tissue engineering, biomedical equipment, and beyond (as shown in Table 2).

The application-based modeling and simulation approach can be used as a systematic optimization tool through the implementation of closed-loop iterative routines for the numerical study of inverse-design complications of the stimuliresponsive and shape-changing $4 \mathrm{D}$ materials. The voxel-based modeling and simulation approach have a few limitations such as those in its current settings, where the object was supposed to have the same size as that of the voxels. Thus, the scope always lies for the voxelized adaption (having a variable voxel size) of the modeling approach, as per the requirement of reducing the computational efforts. While modeling, it is also required to include the effects of collision and gravity, as well as friction. Researchers should also think about the modeling of such smart materials with shape memory alloys to bring the stimuli-responsive properties (such as sensitivity towards heat and light) efficiently and effectively.

The research areas in the $4 \mathrm{D}$ printing can be categorized in various groups, as shown in Fig. 8. Researchers need to explore ways to print such smart 4D materials based on their responses to stimuli, mechanisms of deformation, and mathematical modeling.

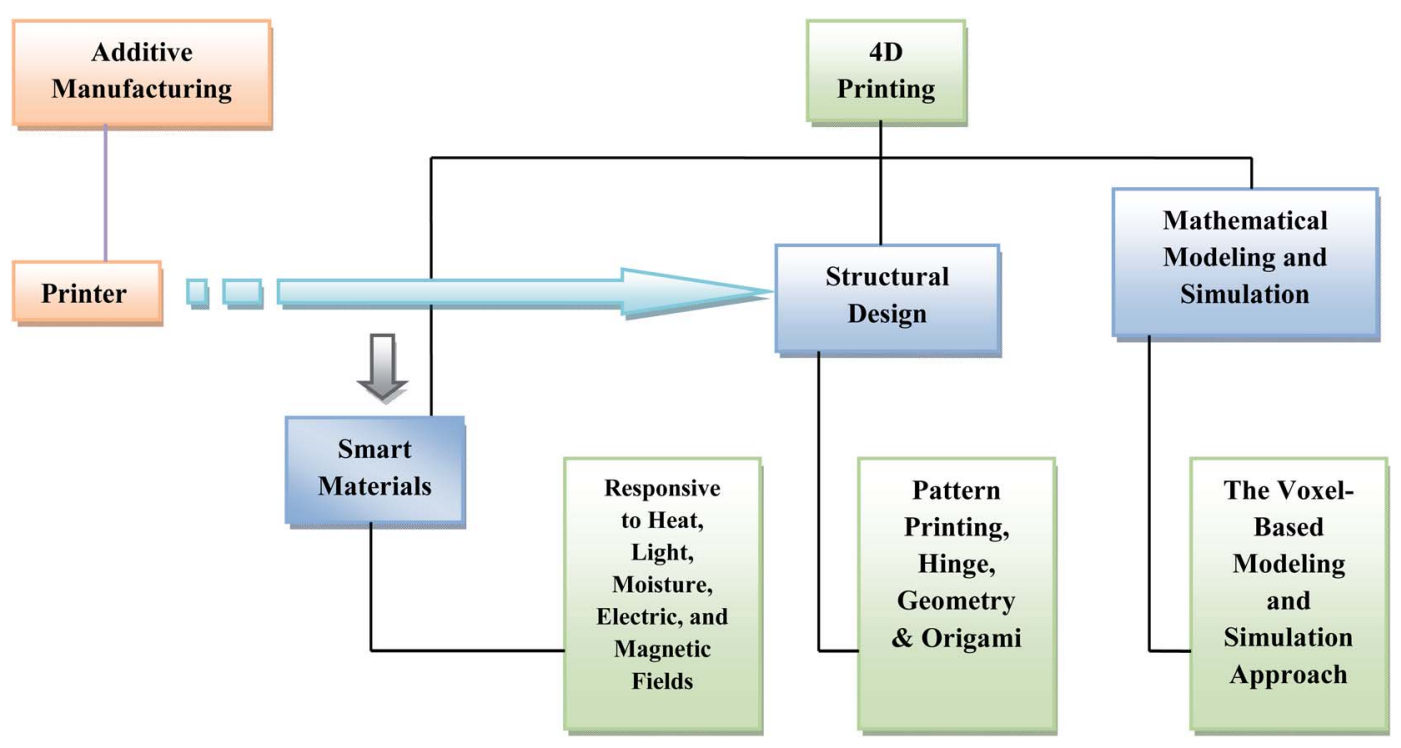

Fig. 8 Research scope in 4D printing. 


\section{Conclusions}

The application-based modeling and simulation approach (based on thin plates and shells having discrete shapes) for phytomimetic (nonlinear elastic) bilayer materials could be used to investigate the residual stresses and strains through the study of the equilibrium sequences of the $4 \mathrm{D}$ printed smart materials that will come in the range of their original and finally restored shapes (Section 2.1). The voxel-based modeling and simulation method is reviewed to motivate researchers while making a choice for additive manufacturing (which has the capability to deal with material complexity). In the voxel-based modeling framework, the basic computations are supposed to be done while underlying the frame, which was formed by the beams connecting the voxel centers (Section 2.2). The degrees of freedom of nodes (in the simple model of $4 \mathrm{D}$ smart material having internal forces responsible for changing its length) were calculated to map the deformation of each beam, while the voxels were morphed using computer graphics. The model was assigned with the properties of piezoelectric, electrostrictive, magnetostrictive, photostrictive, and hydrogel materials to serve the purpose of an intelligent smart material. Sossou et al. (2019) have validated the FEA simulation results with existing experimental data sets. The bi-exponential expressions can be inculcated in the voxel-based modeling and simulation approach to encode the shape changing features of the bioinspired 4DP material, as the shape-changing behavior of such material is a function of time (Section 2.3).

The $4 \mathrm{D}$ printing manufacturing techniques discussed in the fourth case study (Section 2.4) was space and time-dependent. The hydrogel composite ink has a potential to be extended to an advanced level, i.e., liquid-crystal elastomers to anisotropic fillers, such as metallic nanorods to produce biomimetic 4D materials with tunable functionality when combined with flowinduced anisotropy. The smart and novel biocompatible scaffolds (Section 2.5) can be printed after solidification of the soybean epoxidized acrylate resin using the multi-dimensional laser printing technique. This renewable $4 \mathrm{D}$ scaffold material would be a better option to the customary polyethylene glycol diacrylate (PEGDA) to support human bone marrow mesenchymal stem cells (hMSCs), as it has a higher adhesion and proliferation of hMSCs. The biomimetic $4 \mathrm{D}$ printed dual-gel tube (Section 2.5) was found to be capable of changing its shape to pick and drop the cube when operated at different temperatures. Thus, it could serve the purpose of vascular implants (suitable to the growth of tissues) and perform well in soft-robotic endoscopic applications.

\section{Author(s) contributions statements}

Nand Jee Kanu, Research Scholar, S. V. National Institute of Technology, Surat, India (An Institute of National Importance), is pursuing a Ph.D. in Mechanical Engineering and has reviewed and compiled the work. Eva Gupta, Research Scholar, Amity University, Uttar Pradesh, India (An Institute of National Importance), is pursuing a Ph.D. in Electrical Engineering and has reviewed papers on the topic above. Umesh Kumar Vates,
Associate Professor, Amity University, Uttar Pradesh, India, has completed his $\mathrm{PhD}$ in Mechanical engineering from IIT (ISM) Dhanbad, India (An Institute of National Importance). His role is as expert in the work, while monitoring and motivating the abovementioned Ph.D. students. Gyanendra Kumar Singh, Faculty, Federal Technical and Vocational Education and Training Institute, Addis Ababa, Ethiopia, has completed his Ph.D. in Mechanical engineering from M. N. National Institute of Technology, Allahabad, India (An Institute of National Importance). His role is as expert in the work, while monitoring and motivating the abovementioned Ph.D. students.

\section{Conflicts of interest}

The author(s) declare no competing interests.

\section{Acknowledgements}

Nand Jee Kanu, Research Scholar, S. V. National Institute of Technology, Surat, India; Eva Gupta, Research Scholar, Amity University, Uttar Pradesh, India; Umesh Kumar Vates, Associate Professor, Amity University, Uttar Pradesh, India; and Gyanendra Kumar Singh, Faculty, Federal Technical and Vocational Education and Training Institute, Addis Ababa, Ethiopia; have not been funded in any way to carry out the research activities.

\section{References}

1 S. Tibbits, The emergence of " $4 D$ printing", TED Talk (Online), available at https://www.ted.com/talks/skylar_tibbits_the emergence_of_4d_printing, accessed 2019 August 05.

2 F. Momeni and J. Ni, Laws of 4D printing, 2018, arXiv:1810.10376v1 [physics.app-ph].

3 L. Koch, A. Deiwick and B. Chichkov, Laser-Based Cell Printing, $3 D$ Printing and Biofabrication, Springer International Publishing, Cham, 2018, pp. 303-329.

4 Z. Zhang, K. G. Demir and G. X. Gu, Developments in 4Dprinting: a review on current smart materials, technologies, and applications, Int. J. Smart Nano Mater., 2019, 10, 205-224.

5 M. D. Hager, S. Bode, C. Weber and U. S. Schubert, Shape memory polymers: Past, present and future developments, Prog. Polym. Sci., 2015, 49-50, 3-33.

6 Y. Zhou, W. M. Huang, S. F. Kang, X. L. Wu, H. B. Lu, J. Fu and H. Cui, From 3D to 4D printing: Approaches and typical applications, J. Mech. Sci. Technol., 2015, 29, 4281-4288.

7 F. Momeni, S. Sabzpoushan, R. Valizadeh, M. R. Morad, X. Liu and J. Ni, Plant leaf-mimetic smart wind turbine blades by 4D printing, Renewable Energy, 2019, 130, 329-351.

8 Y. Y. C. Choong, S. Maleksaeedi, H. Eng, J. Wei and P. C. Su, $4 \mathrm{D}$ printing of high performance shape memory polymer using stereolithography, Mater. Des., 2017, 126, 219-225.

9 Y. Hu, G. Wu, T. Lan, J. Zhao, Y. Liu and W. Chen, A graphene-based bimorph structure for design of high performance photoactuators, Adv. Mater., 2015, 27, 78677873. 
10 Y. S. Zhang, K. Yue, J. Aleman, K. Mollazadeh-Moghaddam, S. M. Bakht, J. Yang, W. Jia, V. Dell'Erba, P. Assawes, S. R. Shin, M. R. Dokmeci, R. Oklu and A. Khademhosseini, 3D bioprinting for tissue and organ fabrication, Ann. Biomed. Eng., 2017, 45, 148-163.

11 Y. Mao, Z. Ding, C. Yuan, S. Ai, M. Isakov, J. Wu, T. Wang, M. L. Dunn and H. J. Qi, 3D printed reversible shape changing components with stimuli responsive materials, Sci. Rep., 2016, 6, 1-13.

12 K. Zhang, Moisture-responsive films of cellulose stearoyl esters showing reversible shape transitions, Sci. Rep., 2015, 5, 1-13.

13 J. C. Breger, C. Yoon, R. Xiao, H. R. Kwag, M. O. Wang, J. P. Fisher, T. D. Nguyen and D. H. Gracias, Self-folding thermo-magnetically responsive soft microgrippers, ACS Appl. Mater. Interfaces, 2015, 7, 3398-3405.

14 S. Tibbits, C. McKnelly, C. Olguin, D. Dikovsky and S. Hirsch, 4D printing and universal transformation, Proceedings of the 34th annual conference of the Association for Computer Aided Design in Architecture, 2014, pp. 539-548.

15 Y. Liu, B. Shaw, M. D. Dickey and J. Genzer, Sequential selffolding of polymer sheets, Sci. Adv., 2017, 3, 1-8.

16 O. Kuksenok and A. C. Balazs, Stimuli-responsive behavior of composites integrating thermo-responsive gels with photo-responsive fibers, Mater. Horiz., 2016, 3, 53-62.

17 J. Wu, Z. Zhao, X. Kuang, C. M. Hamel, D. Fang and H. J. Qi, Reversible shape change structures by grayscale pattern $4 \mathrm{D}$ printing, Multifunct. Mater., 2018, 1, 15002.

18 A. Miriyev, K. Stack and H. Lipson, Soft material for soft actuators, Nat. Commun., 2017, 8, 1-8.

19 H. Okuzaki, T. Kuwabara, K. Funasaka and T. Saido, Humidity-sensitive polypyrrole films for electro-active polymer actuators, Adv. Funct. Mater., 2013, 23, 4400-4407.

20 K. C. Wong, S. M. Kumta, N. V. Geel and J. Demol, One-step reconstruction with a 3D-printed, biomechanically evaluated custom implant after complex pelvic tumor resection, Comput. Aided Surg., 2015, 20, 14-23.

21 M. Mukhtar, T. Kalganova and N. Lesne, Control of 3D printed ambidextrous robot hand actuated by pneumatic artificial muscles, SAI Intell. Syst. Conf., 2015, 290-300.

22 J. Zuniga, D. Katsavelis, J. Peck, J. Stollberg, M. Petrykowski, A. Carson and C. Fernandez, Cyborg beast: A low-cost 3dprinted prosthetic hand for children with upper-limb differences, BMC Res. Notes, 2015, 8, 10.

23 C. Dally, D. Johnson, M. Canon, S. Ritter and K. Mehta, Characteristics of a 3D-printed prosthetic hand for use in developing countries, 2015 IEEE Global Humanitarian Technology Conference (GHTC), Seattle, WA, USA, 2015, pp. 66-70.

24 S. A. Khaled, J. C. Burley, M. R. Alexander, J. Yang and C. J. Roberts, 3D printing of tablets containing multiple drugs with defined release profiles, Int. J. Pharm., 2015, 494, 643-650.

25 A. Azam, K. E. Laflin, M. Jamal, R. Fernandes and D. H. Gracias, Self-folding micropatterned polymeric containers, Biomed. Microdevices, 2011, 13, 51-58.
26 K. Malachowski, J. Breger, H. R. Kwag, M. O. Wang, J. P. Fisher, F. M. Selaru and D. H. Gracias, Stimuliresponsive theragrippers for chemomechanical controlled release, Angew. Chem., Int. Ed., 2014, 53, 8045-8049.

27 A. W. Martinez and E. L. Chaikof, Microfabrication and nanotechnology in stent design, Wiley Interdiscip. Rev.: Nanomed. Nanobiotechnol., 2011, 3, 256-268.

28 H. Wei, Q. Zhang, Y. Yao, L. Liu, Y. Liu and J. Leng, Directwrite fabrication of 4D active shape-changing structures based on a shape memory polymer and its nanocomposite, ACS Appl. Mater. Interfaces, 2017, 9, 876883.

29 R. J. Morrison, S. J. Hollister, M. F. Niedner, M. Ghadimi Mahani, A. H. Park, D. K. Mehta, R. G. Ohye and G. E. Green, Mitigation of tracheobronchomalacia with 3D-printed personalized medical devices in pediatric patients, Sci. Transl. Med., 2015, 7, 285 ra64.

30 S. Miao, W. Zhu, N. J. Castro, M. Nowicki, X. Zhou, H. Cui, J. P. Fisher and L. G. Zhang, 4D printing smart biomedical scaffolds with novel soybean oil epoxidized acrylate, Sci. Rep., 2016, 6, 27226.

31 S. Miao, H. Cui, M. Nowicki, L. Xia, X. Zhou, S.-J. Lee, W. Zhu, K. Sarkar, Z. Zhang and L. G. Zhang, Stereolithographic $4 \mathrm{D}$ bioprinting of multiresponsive architectures for neural engineering, Adv. Biosyst., 2018, 2, 1800101.

32 A. Martinez, C. Weaver, J. Lopez, S. Bhathena, T. Elsasser, M. Miller, T. Moody, E. Unsworth and F. Cuttitta, Regulation of insulin secretion and blood glucose metabolism by adrenomedullin, Endocrinology, 1996, 137, 2626-2632.

33 C. Wang, J. Wang, X. Zhang, S. Yu, D. Wen, Q. Hu, Y. Ye, H. Bomba, X. Hu and Z. Liu, In situ formed reactive oxygen species-responsive scaffold with gemcitabine and checkpoint inhibitor for combination therapy, Sci. Transl. Med., 2018, 10, eaan3682.

34 H.-C. Yeh, T. T. Brown, N. Maruthur, P. Ranasinghe, Z. Berger, Y. D. Suh, L. M. Wilson, E. B. Haberl, J. Brick and E. B. Bass, Comparative effectiveness and safety of methods of insulin delivery and glucose monitoring for diabetes mellitus: a systematic review and meta-analysis, Ann. Intern. Med., 2012, 157, 336-347.

35 J.D.R.F.C.G.M.S. Group, Continuous glucose monitoring and intensive treatment of type 1 diabetes, N. Engl. J. Med., 2008, 359, 1464-1476.

36 J. Yu, Y. Zhang, Y. Ye, R. DiSanto, W. Sun, D. Ranson, F. S. Ligler, J. B. Buse and Z. Gu, Microneedle array patches loaded with hypoxia-sensitive vesicles provide fast glucose-responsive insulin delivery, Proc. Natl. Acad. Sci., 2015, 112, 8260-8265.

37 A. Adams, A. Malkoc and J. T. La Belle, The Development of a Glucose Dehydrogenase 3D-Printed Glucose Sensor: A Proof-of-Concept Study, J. Diabetes Sci. Technol., 2018, 12, 176-182.

38 E. Song, T. H. da Costa and J.-W. Choi, A chemiresistive glucose sensor fabricated by inkjet printing, Microsyst. Technol., 2017, 23, 3505-3511. 
39 A. Matsumoto, T. Ishii, J. Nishida, H. Matsumoto, K. Kataoka and Y. Miyahara, A synthetic approach toward a self-regulated insulin delivery system, Angew. Chem., Int. Ed., 2012, 51, 2124-2128.

40 M. Brownlee and A. Cerami, A glucose-controlled insulindelivery system: semisynthetic insulin bound to lectin, Science, 1979, 206, 1190-1191.

41 K. Podual, F. J. Doyle III and N. A. Peppas, Glucosesensitivity of glucose oxidase-containing cationic copolymer hydrogels having poly(ethylene glycol) grafts, $J$. Controlled Release, 2000, 67, 9-17.

42 J. Song and J. R. Millman, Economic 3D-printing approach for transplantation of human stem cell-derived $\beta$-like cells, Biofabrication, 2016, 9, 015002.

43 T. Hayashi, M. F. Hirshman, E. J. Kurth, W. W. Winder and L. J. Goodyear, Evidence for $5^{\prime}$ AMP-activated protein kinase mediation of the effect of muscle contraction on glucose transport, Diabetes, 1998, 47, 1369-1373.

44 T. Isobe, H. Takahashi, S. Ueki, J. Takagi and Y. Saito, Activity-independent cell adhesion to tissue-type transglutaminase is mediated by $\alpha 4 \beta 1$ integrin, Eur. $J$. Cell Biol., 1999, 78, 876-883.

45 S. Ueki, J. Takagi and Y. Saito, Dual functions of transglutaminase in novel cell adhesion, J. Cell Sci., 1996, 109, 2727-2735.

46 S. P. Galuska, R. Geyer, R. Gerardy-Schahn, M. Mühlenhoff and H. Geyer, Enzyme-dependent variations in the polysialylation of the neural cell adhesion molecule (NCAM) in vivo, J. Biol. Chem., 2008, 283, 17-28.

47 J. R. Tauro and R. A. Gemeinhart, Matrix metalloprotease triggered delivery of cancer chemotherapeutics from hydrogel matrixes, Bioconjugate Chem., 2005, 16, 1133-1139.

48 Q. Hu, P. S. Katti and Z. Gu, Enzyme-responsive nanomaterials for controlled drug delivery, Nanoscale, 2014, 6, 12273-12286.

49 X. Wang, X. H. Qin, C. Hu, A. Terzopoulou, X. Z. Chen, T. Y. Huang, K. Maniura-Weber, S. Pané and B. J. Nelson, 3D Printed Enzymatically Biodegradable Soft Helical Microswimmers, Adv. Funct. Mater., 2018, 28, 1804107.

50 H. Ceylan, I. C. Yasa, O. Yasa, A. F. Tabak, J. Giltinan and M. Sitti, 3D-Printed Biodegradable Microswimmer for Drug Delivery and Targeted Cell Labeling, 2018, bioRxiv, 379024.

51 K. H. Song, C. B. Highley, A. Rouff and J. A. Burdick, Complex 3D-Printed Microchannels within Cell-Degradable Hydrogels, Adv. Funct. Mater., 2018, 28, 1801331.

52 M. Zelzer, S. J. Todd, A. R. Hirst, T. O. McDonald and R. V. Ulijn, Enzyme responsive materials: design strategies and future developments, Biomater. Sci., 2013, 1, 11-39.

53 W. M. van Rees, E. A. Matsumoto, A. Sydney Gladman, J. A. Lewis and L. Mahadevan, Mechanics of biomimetic 4D printed structures, Soft Matter, 2018, 14, 8771-8779.

54 A. S. Gladman, E. A. Matsumoto, R. G. Nuzzo, L. Mahadevan and J. A. Lewis, Biomimetic 4D printing, Nat. Mater., 2016, 15, 413-418.

55 W. M. van Rees, E. Vouga and L. Mahadevan, Growth patterns for shape-shifting elastic bilayers, Proc. Natl. Acad. Sci. U. S. A., 2017, 114, 11597-11602.
56 E. Efrati, E. Sharon and R. Kupferman, Elastic theory of unconstrained non-Euclidean plates, J. Mech. Phys. Solids, 2009, 57, 762-775.

57 C. Weischedel, A. Tuganov, T. Hermansson, J. Linn and M. Wardetzky, Construction of discrete shell models by geometric finite differences, Fraunhofer ITWM Technical Report, 2012, vol. 220, pp. 1-10.

58 J. N. Reddy, Mechanics of Laminated Composite Plates and Shells, CRC Press, 2nd edn, 2004, ISBN 9780849315923.

59 E. Grinspun, Y. Gingold, J. Reisman and D. Zorin, Computing discrete shape operators on general meshes, Comput. Graph. Forum, 2006, 25, 547-556.

60 G. Sossou, F. Demoly, H. Belkebir, H. J. Qi, S. Gomes and G. Montavon, Design for 4D printing: A voxel-based modeling and simulation of smart materials, Mater. Des., 2019, 175, 107798.

61 M. Vaezi, S. Chianrabutra, B. Mellor and S. Yang, Multiple material additive manufacturing - part 1: a review, Virtual Phys. Prototyping, 2013, 8, 19-50.

62 Stratasys, PolyJet Technology for 3D Printing, available at http://www.stratasys.com/polyjet-technology, accessed 2019 August 05.

63 K. Yamaguchi, K. Sakai, T. Yamanaka and T. Hirayama, Generation of three-dimensional micro structure using metal jet, Precis. Eng., 2000, 24, 2-8.

64 S. Tibbits, 4D printing: multi-material shape change, Architect. Des., 2014, 84, 116-121.

65 M. Ferrara and M. Bengisu, Materials that Change Color, PoliMI SpringerBriefs, Springer International Publishing, 2014, p. 139.

66 E. Hristoforou and A. Ktena, Magnetostriction and magnetostrictive materials for sensing applications, $J$. Magn. Magn. Mater., 2007, 316, 372-378.

67 G. I. Peterson, M. B. Larsen, M. A. Ganter, D. W. Storti and A. J. Boydston, 3D-printed mechanochromic materials, ACS Appl. Mater. Interfaces, 2015, 7, 577-583.

68 J. Choi, O.-C. Kwon, W. Jo, H. J. Lee and M.-W. Moon, 4D printing technology: A Review, $3 D$ Print. Addit. Manuf., 2015, 2, 159-167.

69 S. K. Leist and J. Zhou, Current status of $4 \mathrm{D}$ printing technology and the potential of light reactive smart materials as 4D printable materials, Virtual Phys. Prototyping, 2016, 11, 249-262.

70 X. K. Zhong, E. M. T. Joanne, L. Yong, K. C. Chee, Y. Shoufeng, A. Jia, L. K. Fai and Y. W. Yee, 3D printing of smart materials: a review on recent progresses in $4 \mathrm{D}$ printing, Virtual Phys. Prototyping, 2015, 10, 103-122.

71 F. Momeni, S. M.Mehdi Hassani. N, X. Liu and J. Ni, A review of 4D printing, Mater. Des., 2017, 122, 42-79.

72 M. C. Mulakkal, R. S. Trask, V. P. Ting and A. M. Seddon, Responsive cellulose-hydrogel composite ink for $4 \mathrm{D}$ printing, Mater. Des., 2018, 160, 108-118.

73 A. B. Baker, S. R. G. Bates, T. M. Llewellyn-Jones, L. P. B. Valori, M. P. M. Dicker and R. S. Trask, 4D printing with robust thermoplastic polyurethane hydrogel-elastomer trilayers, Mater. Des., 2019, 163, 107544. 
74 M. Okereke and S. Keates, Direct Stiffness Method, Finite Element Applications: A Practical Guide to the FEM Process, Springer International Publishing, Cham, 2018, pp. 47-106.

75 K. D. Hjelmstad, The linear theory of beams, Fundamentals of Structural Mechanics, Springer US, Boston, MA, 2005, pp. 241-291.

76 M. Bodaghi, A. R. Damanpack and W. H. Liao, Adaptive metamaterials by functionally graded 4D printing, Mater. Des., 2017, 135, 26-36.

77 M. Bodaghi, A. R. Damanpack and W. H. Liao, Selfexpanding/shrinking structures by 4D printing, Smart Mater. Struct., 2016, 25, 105034.

78 M. Bodaghi, A. R. Damanpack and W. H. Liao, Triple shape memory polymers by $4 \mathrm{D}$ printing, Smart Mater. Struct., 2018, 27, 065010.

79 Q. Ge, A. H. Sakhaei, H. Lee, C. K. Dunn, N. X. Fang and M. L. Dunn, Multimaterial 4D printing with tailorable shape memory polymers, Sci. Rep., 2016, 6, 31110.

80 Noumenon, The Material is the Mechanism, available at http://noumenon.eu/\#\&panel1-12, accessed 2018 March 21.

81 K. K. Westbrook and H. J. Qi, Actuator designs using environmentally responsive hydrogels, J. Intell. Mater. Syst. Struct., 2008, 19, 597-607.

82 E. Lefebvre, A. Piselli, F. Jenny, D. Delafosse \& B. Del Curto, Smart materials: Development of new sensory experiences through stimuli responsive materials, 5th STS Italia Conference A Matter of Design: Making Society through Science and Technology, STS Italia, Milan, Italy, 2014, pp. 367-382.

83 R. E. Pelrine, R. D. Kornbluh and J. P. Joseph, Electrostriction of polymer dielectrics with compliant electrodes as a means of actuation, Sens. Actuators, A, 1998, 64, 77-85.

84 Q. Zhao, H. J. Qi and T. Xie, Recent progress in shape memory polymer: new behavior, enabling materials, and mechanistic understanding, Prog. Polym. Sci., 2015, 49-50, 79-120.

85 J. M. Jani, M. Leary, A. Subic and M. A. Gibson, A review of shape memory alloy research, applications and opportunities, Mater. Des., 2014, 56, 1078-1113.

86 G. Ravichandran, Active materials, in Springer Handbook of Experimental Solid Mechanics, ed. N. W. Sharpe, Springer US, Boston, MA, 2008, pp. 159-168.

87 L. Eyraud, P. Eyraud, P. Gonnard and M. Troccaz, Matériaux électrostrictifs pour actuateurs, Rev. Phys. Appl., 1988, 23, 879-889.

88 B. Kundys, Photostrictive materials, Appl. Phys. Rev., 2015, 2, 011301.

89 H. Finkelmann, E. Nishikawa, G. G. Pereira and M. Warner, A new opto-mechanical effect in solids, Phys. Rev. Lett., 2001, 87, 015501.

90 Y. Zhou, L. You, S. Wang, Z. Ku, H. Fan, D. Schmidt, A. Rusydi, L. Chang, L. Wang, P. Ren, L. Chen, G. Yuan, L. Chen and J. Wang, Giant photostriction in organicinorganic lead halide perovskites, Nat. Commun., 2016, 7, 11193.
91 S. E. Bakarich, R. Gorkin III, M. Panhuis and G. M. Spinks, 4D printing with mechanically robust, thermally actuating hydrogels, Macromol. Rapid Commun., 2015, 36, 1211-1217.

92 A. Pytel and J. Kiusalaas, Mechanics of materials, Cengage Learning, Stamford, USA, 2nd edn, 2012, ISBN-13: 978-0495-66775-9.

93 M. Kaviany, Essentials of heat transfer: principles, materials, and applications. Cambridge University Press, 2011, ISBN: 9780511998195.

94 B. Kim, K. La Flamme and N. A. Peppas, Dynamic swelling behavior of $\mathrm{pH}$-sensitive anionic hydrogels used for protein delivery, J. Appl. Polym. Sci., 2003, 89, 1606-1613.

95 A. R. Berens and H. B. Hopfenberg, Diffusion and relaxation in glassy polymer powders: 2 . Separation of diffusion and relaxation parameters, Polymer, 1978, 19, 489-496.

96 M. Czugala, C. O'Connell, C. Blin, P. Fischer, K. J. Fraser, F. Benito-Lopez and D. Diamond, Swelling and shrinking behavior of photoresponsive phosphonium-based ionogel microstructures, Sens. Actuators, B, 2014, 194, 105-113.

97 Q. Ge, H. J. Qi and M. L. Dunn, Active materials by fourdimension printing, Appl. Phys. Lett., 2013, 103, 131901.

98 D. Ratna and J. Karger-Kocsis, Recent advances in shape memory polymers and composites: a review, J. Mater. Sci., 2008, 43, 254-260.

99 R. M. Erb, J. S. Sander, R. Grisch and A. E. R. Studart, Selfshaping composites with programmable bioinspired microstructures, Nat. Commun, 2012, 4, 1712.

100 H. Thérien-Aubin, Z. L. Wu, Z. Nie and E. Kumacheva, Multiple shape transformations of composite hydrogel sheets, J. Am. Chem. Soc., 2013, 125, 4834-4839.

101 S. Armon, E. Efrati, R. Kupferman and E. Sharon, Geometry and mechanics in the opening of chiral seed pods, Science, 2011, 333, 1726-1730.

102 H. Aharoni, E. Sharon and R. Kupferman, Geometry of thin nematic elastomer sheets, Phys. Rev. Lett., 2014, 113, 257801.

103 F. P. Melchels, J. Feijen and D. W. Grijpma, A review on stereolithography and its applications in biomedical engineering, Biomaterials, 2010, 31, 6121-6130.

104 S. Miao, P. Wang, Z. Su and S. Zhang, Vegetable-oil-based polymers as future polymeric biomaterials, Acta Biomater., 2014, 10, 1692-1704.

105 S. Miao, L. Sun, P. Wang, R. Liu, Z. Su and S. Zhang, Soybean oil-based polyurethane networks as candidate biomaterials: Synthesis and biocompatibility, Eur. J. Lipid Sci. Technol., 2012, 114, 1165-1174.

106 T. F. Garrison, M. R. Kessler and R. C. Larock, Effects of unsaturation and different ring-opening methods on the properties of vegetable oil-based polyurethane coatings, Polymer, 2014, 55, 1004-1011.

107 G. Lligadas, J. C. Ronda, M. Galia and V. Cadiz, Renewable polymeric materials from vegetable oils: a perspective, Mater. Today, 2013, 16, 337-343.

108 Q. Ge, C. K. Dunn, H. J. Qi and M. L. Dunn, Active origami by 4D printing, Smart Mater. Struct., 2014, 23, 094007.

109 N. J. Castro, J. O'Brien and L. G. Zhang, Integrating biologically inspired nanomaterials and table-top 
stereolithography for 3D printed biomimetic osteochondral scaffolds, Nanoscale, 2015, 7, 14010-14022.

110 F. P. Melchels, J. Feijen and D. W. Grijpma, A poly(D,Llactide) resin for the preparation of tissue engineering scaffolds by stereolithography, Biomaterials, 2009, 30, 3801-3809.

111 D. W. Grijpma, Q. Hou and J. Feijen, Preparation of biodegradable networks by photo-crosslinking lactide, $\varepsilon^{-}$ caprolactone and trimethylene carbonate-based oligomers functionalized with fumaric acid monoethyl ester, Biomaterials, 2005, 26, 2795-2802.

112 J. Jansen, F. P. Melchels, D. W. Grijpma and J. Feijen, Fumaric acid monoethyl ester-functionalized poly (D,Llactide) $/ N$-vinyl-2-pyrrolidone resins for the preparation of tissue engineering scaffolds by stereolithography, Biomacromolecules, 2008, 10, 214-220.

113 M. N. Cooke, J. P. Fisher, D. Dean, C. Rimnac and A. G. Mikos, Use of stereolithography to manufacture critical-sized 3D biodegradable scaffolds for bone ingrowth, J. Biomed. Mater. Res., Part B, 2003, 64, 65-69.

114 S. Miao, N. Callow, P. Wang, Y. Liu, Z. Su and S. Zhang, Soybean oil-based polyurethane networks: shape-memory effects and surface morphologies, J. Am. Oil Chem. Soc., 2013, 90, 1415-1421.

115 S. Miao, P. Wang, Z. Su, Y. Liu and S. Zhang, Soybean oil-based shape-memory polyurethanes: Synthesis and characterization, Eur. J. Lipid Sci. Technol., 2012, 114, 1345-1351.

116 B. Gao, Q. Yang, X. Zhao, G. Jin, Y. Ma and F. Xu, 4D Bioprinting for Biomedical Applications, Trends Biotechnol., 2016, 34, 746-756.

117 J. Liu, O. Erol, A. Pantula, W. Liu, Z. Jiang, K. Kobayashi, D. Chatterjee, N. Hibino, L. H. Romer, S. H. Kang, T. D. Nguyen and D. H. Gracias, Dual-gel 4D Printing of Bioinspired Tubes, ACS Appl. Mater. Interfaces, 2019, 11, 8492-8498.

118 M. Zarek, N. Mansour, S. Shapira and D. Cohn, 4D Printing of Shape Memory-Based Personalized Endoluminal Medical Devices, Macromol. Rapid Commun., 2016, 38, 1600628.

119 S. Miao, N. Castro, M. Nowicki, L. Xia, H. Cui, X. Zhou, W. Zhu, S. J. Lee, K. Sarkar, G. Vozzi, Y. Tabata, J. Fisher and L. G. Zhang, 4D printing of polymeric materials for tissue and organ regeneration, Mater. today, 2017, 20, 577-591.

120 S. Saunders, $4 D$ printing technique could be used to develop $3 D$ printed human organs for transplant patients, available at https://3dprint.com/196141/4d-printing-humanorgans/, accessed 2017 December 04.

121 S. Akbari, A. H. Sakhaeim, K. Kowsari, B. Yang, A. Serjouei, Z. Yuanfang and Q. Ge, Enhanced multi-material 4D printing with active hinges, Smart Mater. Struct., 2018, 27, 1-23.
122 I. U. Haq, $4 D$ Printed Implant Saved Babies with Breathing Problems, available at http://computingcage.com/4dimplant-saves-babies-with-breathing-problems/, accessed 2015 November 26.

123 C. Q. Choi, 4D Implant Saves Babies with Breathing Problems, available at https:/www.livescience.com/50668-4d-implantbabies-breathing-problems.html, accessed 2015 April 29.

$124 \mathrm{~W}$. J. Hendrikson, J. Rouwkema, F. Clementi, C. A. Blitterswijk, S. Fare and L. Moroni, Towards 4D printed scaffolds for tissue engineering: exploiting $3 \mathrm{D}$ shape memory polymers to deliver time-controlled stimulus on cultured cells, Biofabrication, 2017, 9, 031001.

125 C. A. Mandon, L. J. Blum and C. A. Marquette, 3D-4D Printed Objects: New Bioactive Material Opportunities, Micromachines, 2017, 8, 102.

126 H. G. Yi, H. Lee and D. W. Cho, 3D Printing of Organs-OnChips, Bioengineering, 2017, 4, 10.

127 J. Gosnell, T. Pietila, B. P. Samuel, H. K. N. Kurup, M. P. Haw and J. J. Vettukattil, Integration of computed tomography and three-dimensional echocardiography for hybrid three dimensional printing in congenital heart disease, J. Digital Imaging, 2016, 29, 665-669.

128 P. He, J. Zhao, J. Zhang, B. Li, Z. Gou, M. Gou and X. Li, Bioprinting of skin constructs for wound healing, Burns Trauma, 2018, 6, 1-10.

129 X. Kuang, K. Chen, C. K. Dunn, J. Wu, V. C. F. Li and H. J. Qi, 3D Printing of Highly Stretchable, ShapeMemory, and Self-Healing Elastomer toward Novel 4D Printing, ACS Appl. Mater. Interfaces, 2018, 10, 7381-7388.

130 E. Pei and G. H. Loh, Technological considerations for 4D printing: an overview, Prog. Addit. Manuf., 2018, 3, 95-107.

131 N. J. Castro, C. Meinert, P. Levett and D. W. Hutmacher, Current developments in multifunctional smart materials for 3D/4D bioprinting, Curr. Opin. Biomed. Eng., 2017, 2, 67-75.

132 T. Zhao, R. Yu, X. Li, B. Cheng, Y. Zhang, X. Yang, X. Zhao, Y. Zhao and W. Huang, 4D printing of shape memory polyurethane via stereolithography, Eur. Polym. J., 2018, 101, 120-126.

133 M. P. Chae, D. J. Hunter-Smith, I. De-Silva, S. Tham, R. T. Spychal and W. M. Rozen, Four-Dimensional (4D) Printing: A New Evolution in Computed TomographyGuided Stereolithographic Modeling. Principles and Application, J. Reconstr. Microsurg., 2015, 31, 458-463.

134 A. Y. Lee, J. An and C. K. Chua, Two-Way 4D Printing: A Review on the Reversibility of 3D-Printed Shape Memory Materials, Engineering, 2017, 3, 663-674.

135 M. Javaid, A. Haleem and L. Kumar, Current status and applications of 3D scanning in dentistry, Clin. Epidemiol. Global Health, 2019, 7, 228-233.

$136 \mathrm{H}$. Sanjeet and H. Albert, Improving the Fontan: Presurgical planning using Four-Dimensional (4D) Flow, Biomechanical Modeling and Three Dimensional (3D) Printing, Prog. Pediatr. Cardiol., 2016, 43, 57-60. 